\title{
Helicopter Tail Rotor Thrust and Main Rotor Wake Coupling in Crosswind Flight
}

\author{
Timothy M. Fletcher* and Richard E. Brown‡ \\ University of Glasgow, Glasgow, Scotland G12 8QQ, United Kingdom
}

DOI: $10.2514 / 1 . C 031018$

\begin{abstract}
The tail rotor of a helicopter with a single main rotor configuration can experience a significant reduction in thrust when the aircraft operates in crosswind flight. Brown's vorticity transport model has been used to simulate a main rotor and tail rotor system translating at a sideslip angle that causes the tail rotor to interact with the main rotor tip vortices as they propagate downstream at the lateral extremities of the wake. The tail rotor is shown to exhibit a distinct directionally dependent mode during which tail rotors that are configured so that the blades travel forward at the top of the disk develop less thrust than tail rotors with the reverse sense of rotation. The range of flight speeds over which this mode exists is shown to vary considerably with the vertical location of the tail rotor. At low flight speeds, the directionally dependent mode occurs because the tail rotor is immersed within not only the downwash from the main rotor but also the rotational flow associated with clusters of largely disorganized vorticity within the main rotor wake. At higher flight speeds, however, the tail rotor is immersed within a coherent supervortex that strongly influences the velocity field surrounding the tail rotor.
\end{abstract}

\section{Nomenclature}

$A=$ rotor disk area

$C_{T}=$ rotor thrust coefficient, $T / \rho A(\Omega R)^{2}$

$C_{z}=$ blade loading, scaled by $\rho c(\Omega R)^{2} / 2$

$c \quad=$ blade chord

iso $=$ isolated tail rotor

$R=$ rotor radius

$r=$ radial ordinate on blade

$S=$ vorticity source

$T=$ rotor thrust

$T^{*}=$ tail rotor thrust ratio, equal to rotor thrust scaled by isolated rotor thrust in hover

$t=$ tail rotor

$U=$ resultant sectional velocity, scaled by $\Omega R$

$u=$ flow velocity

$u_{b}=$ flow velocity relative to the blade

$\alpha=$ angle of attack

$\theta_{0}=$ collective pitch

$\mu=$ advance ratio (flight speed, scaled by $\Omega R$ )

$v=$ viscosity

$\rho=$ density

$\Omega=$ rotor rotational speed

$\omega=$ vorticity

$\omega_{b}=$ bound vorticity

\section{Introduction}

$\mathbf{T}$ HE thrust that is developed by the tail rotor of a helicopter with a single main rotor configuration is known to be influenced strongly, in certain flight conditions, by the sense of rotation of the tail rotor $[1,2]$. If a helicopter with a single main rotor translates in crosswind flight at a high angle of sideslip (typically in the range of $50-70^{\circ}$ ), the wake that is induced by the main rotor of the helicopter

Received 10 March 2010; revision received 19 May 2010; accepted for publication 22 May 2010. Copyright () 2010 by Timothy M. Fletcher and Richard E. Brown. Published by the American Institute of Aeronautics and Astronautics, Inc., with permission. Copies of this paper may be made for personal or internal use, on condition that the copier pay the $\$ 10.00$ per-copy fee to the Copyright Clearance Center, Inc., 222 Rosewood Drive, Danvers, MA 01923; include the code 0021-8669/10 and $\$ 10.00$ in correspondence with the CCC.

*Postdoctoral Research Assistant, Department of Aerospace Engineering; t.fletcher@eng.gla.ac.uk. Member AIAA.

${ }^{\dagger}$ Mechan Professor of Engineering, Department of Aerospace Engineering; rbrown@aero.gla.ac.uk. Member AIAA. can impinge directly on the tail rotor, thereby establishing a coupling between the thrust that is developed by the tail rotor and the highly nonuniform flow environment in which it operates. When the helicopter operates at high angles of sideslip, within a narrow range of flight speeds, a tail rotor that is configured so that the blades travel forward at the top of the disk (termed top-forward tail rotors) experiences a reduction in thrust compared with that of the tail rotor when operating in isolation from the main rotor, if the collective pitch setting is identical in both cases. In contrast, those tail rotors with the opposite sense of rotation (termed top-aft tail rotors) can experience an increase in thrust compared with the isolated tail rotor. Whilst any absence of equilibrium in the yaw moment on the helicopter would be routinely corrected by the pilots during flight, the severity of the main rotor-tail rotor (MR-TR) interaction that is experienced at high angles of sideslip can severely curtail the margin that is available for controlling the tail rotor collective pitch and, therefore, the ability of the pilot to control the yaw attitude and rate of the helicopter [3].

The fact that top-forward tail rotors can produce less thrust at a given collective pitch setting compared with tail rotors with the topaft sense of rotation is sometimes referred to as a "loss of tail rotor effectiveness". A helicopter with a main rotor that rotates counterclockwise when viewed from above requires a tail rotor that produces a force to starboard in order to counteract the torque reaction of the main rotor on the fuselage. There are several possible physical situations when a loss of tail rotor effectiveness might occur. When such a helicopter translates directly to port over a narrow range of flight speeds, typically found to be in the range $30-50 \mathrm{kt}$, or is hovering in an equivalent crosswind, the tail rotor operates effectively in descending flight and may enter a flow regime known as the vortex ring state. When operating within the vortex ring state, the flow through the tail rotor is characterized by significant recirculation during which the rotor loading is highly unsteady. The operation of the tail rotor within the vortex ring state can significantly impair the directional control of the helicopter, and it is distinct from the degradation of helicopter controllability that is caused by aerodynamic interaction between the main and tail rotors [4, 5$]$. Furthermore, the presence of a large ground vortex toward the rear of the helicopter when it translates directly aft in ground effect has been shown to impair the directional control of helicopters with a single main rotor configuration [6]. The term loss of tail rotor effectiveness is thought, therefore, to be ambiguous within the present context and, although it has been used within the literature to describe a variety of phenomena in which the control and aerodynamic performance of the tail rotor are coupled, it will not be used within the remainder of this paper.

In 1993, Ellin performed flight tests using a Lynx helicopter translating in $60^{\circ}$ sideslip to starboard. These tests demonstrated that 
there is significant variability in the thrust that is developed by a topforward tail rotor over a narrow range of low to moderate flight speeds [7]. In an attempt to better understand the aerodynamic process that governs the variability in thrust, Ellin used pressure taps to measure the unsteady characteristics of the leading-edge pressure coefficient along the tail rotor blades. It was found that there was no significant variation in the loading on the tail rotor blades at the blade passage frequency of the main rotor, as would be expected if the blades were interacting with the concentrated vortices that are trailed from the tips of the main rotor blades. Instead, the tail rotor was thought to interact with the bundled supervortices that are known to be formed by the coalescence of these tip vortices at the lateral extremities of the main rotor wake as the helicopter translates at moderate to high advance ratios [4]. Importantly, the coalescence of the tip vortices into larger and more coherent supervortices smears much of the temporal variability in the velocity field near to the tail rotor that would otherwise occur at the blade passage frequency of the main rotor.

Ellin's assertion [7] that the variability in tail rotor thrust is caused by the impingement of the supervortices on the tail rotor was neither confirmed nor refuted by later wind-tunnel experiments that were conducted on a helicopter with a single main rotor and a tail rotor as part of the HELIFLOW project [8]. The helicopter model that was used during the HELIFLOW project consisted of the four-bladed main rotor rig that was operated at that time by the U. K. Defence Evaluation and Research Establishment [9], and a four-bladed tail rotor that was mounted on a traverse that allowed the sideslip angle of the rotor system to be varied. In the past, it had been shown that, in forward flight at high angles of sideslip, the vertical location of the tail rotor could strongly influence the thrust that the tail rotor produces [10]. The HELIFLOW experiments were designed, therefore, to demonstrate how the performance of the tail rotor varies with both the sense of rotation of the rotor and its vertical position. Figure 1 shows the measured thrust that was developed by the HELIFLOW tail rotor as a function of the overall advance ratio of the helicopter, while the tail rotor was operated with a fixed collective pitch. Four different combinations of tail rotor vertical location and sense of rotation (top-aft and top-forward) are represented in the figure. The thrust data that are shown in Fig. 1 are scaled by the quasisteady thrust that is developed by the same tail rotor when operating in hover at the same collective pitch setting but in isolation from the main rotor. Figure 1 clearly indicates that the thrust that is developed by the tail rotor is sensitive to its direction of rotation. At very low advance ratios (less than approximately 0.05 ), the thrust that is developed by the tail rotor appears to be largely independent of its sense of rotation. If the advance ratio of the helicopter is increased, however, a region of the flight envelope is encountered in which the thrust that is developed by the top-aft tail rotor is substantially greater than that of the top-forward tail rotor. In addition, Fig. 1 shows that, by raising the tail rotor from a relatively low position to a location at which its hub was at the same height as the hub of the main rotor, the divergence between the thrust that was developed by the tail rotors with opposing senses of rotation was delayed to a significantly higher advance ratio.

The insights that are provided by both Ellin's work [7] and the HELIFLOW project contribute significantly to our understanding of how the aerodynamic interaction between the main and tail rotors of a conventional helicopter influences the performance of the tail rotor in forward flight at high angles of sideslip. Unfortunately, the existing studies of MR-TR interaction in crosswind flight have addressed only a limited set of helicopter configurations and operating conditions and, consequently, the influence that the vertical location and the sense of rotation of the tail rotor has on its performance has been only partially characterized. Equally as important, the variation with advance ratio of the unsteadiness and rotation within flow that surrounds the tail rotor remains poorly understood. This paper will show how a computational code that is known as the vorticity transport model (VTM) can be used to simulate the aerodynamic performance of a generic tail rotor in $60^{\circ}$ sideslip by capturing to a high fidelity the development of the wake that is induced by the main rotor. Importantly, aerodynamic simulation using the VTM allows the helicopter model to be adapted with relative ease so that a wide range of different configurations and flight conditions can be represented. The objectives of this paper are to better understand the extent of the region of the flight envelope in which the performance of the tail rotor is governed by its sense of rotation, to provide guidance to helicopter designers as to how the location of this region within the flight envelope varies with the vertical location of the tail rotor, and to provide a clearer characterization of the aerodynamic mechanism by which the thrust of the tail rotor is coupled to the structure and the dynamics of the main rotor wake.

\section{Helicopter Model}

A simplified model that is composed of only the main rotor and the tail rotor of a conventional helicopter was simulated using the VTM, developed by Brown [11] and Brown and Line [12]. The VTM couples an efficient model of the blade aerodynamics that is based on an extension of the lifting-line theory to a model for the flow surrounding the rotor system (or wake) that solves the unsteady Navier-Stokes equation in vorticity-velocity form. On making the physically realistic assumption of incompressibility within the wake, the Navier-Stokes equations are recast as the vorticity transport equation:

$$
\frac{\partial}{\partial t} \omega+u \cdot \nabla \omega-\omega \cdot \nabla u=S+v \nabla^{2} \omega
$$

Equation (1) is discretized in finite-volume form using a Cartesian mesh within the domain surrounding the MR-TR system. The advection, stretching, and diffusion terms within the vorticity transport equation describe the changes in the vorticity field $\omega$ with

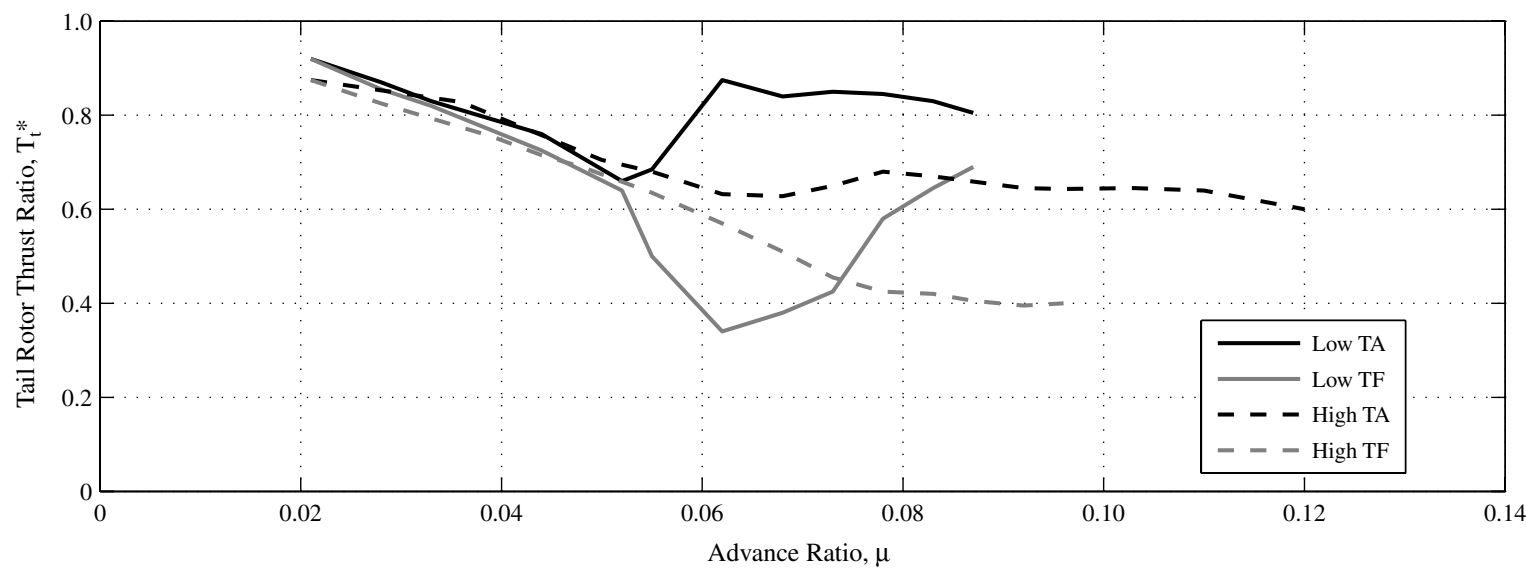

Fig. 1 Measurements made during the HELIFLOW experiments of the thrust developed by tail rotors in four different configurations (low/high: relative vertical locations of the tail rotor, TA: top-aft tail rotor, TF: top-forward tail rotor). (Data sourced from Kaynes et al. [8].) 
time and space as a function of the velocity field $u$ and the viscosity $v$. The Reynolds number of the flow features within the wake is sufficiently high that the structure and dynamics of the wake, at the scales of significance for the physics of MR-TR interaction, are governed almost entirely by inertial processes; therefore, Eq. (1) is solved in inviscid form.

As the flowfield surrounding the MR-TR system is advanced through time, the vorticity field is coupled to the velocity field by using a fast multipole method to solve the Biot-Savart law, which may be stated in differential form as

$$
\nabla^{2} u=-\nabla \times \omega
$$

Mass conservation is achieved to numerical accuracy by using a kernel within the fast multipole method that is divergence free. Use of the fast multipole method, in conjunction with an adaptive grid in which cells are only present within the calculation when the vorticity within them is nonzero, dramatically increases the computational efficiency of the scheme and renders it effectively boundary free under the assumption that there is zero vorticity outside the computational domain. The numerical diffusion (or smearing) of vorticity within the flowfield surrounding the rotorcraft is kept at a very low level by using a convection algorithm that is based on the weighted average flux method that was developed by Toro [13].

The vorticity that is created at the surfaces of the blades as they develop lift is accounted for by including a vorticity source term, $S$, on the right-hand side of Eq. (1). The vorticity source term is computed as the sum of the temporal and spatial variations in the bound vorticity $\omega_{b}$ on the rotor blades:

$$
S=-\frac{\mathrm{d}}{\mathrm{d} t} \omega_{b}+u_{b} \nabla \cdot \omega_{b}
$$

The distribution of bound vorticity on the blades is determined using an extension of the lifting-line theory that has been appropriately modified by the use of two-dimensional experimental measurements to ensure that the real performance of a given distribution of airfoils along the length of the blade is represented. VTM predictions of rotor aerodynamic performance and wake dynamics have been analyzed extensively in the past, and they have been verified within the context of coaxial helicopter rotors [14] and helicopter rotor-fuselage aerodynamic interactions [15]. The VTM has also been used successfully to predict the aerodynamic performance and acoustic signature of complex helicopter configurations that involve multiple rotor-rotor and rotor-airframe interactions [16].

A series of simulations were performed by accelerating the MRTR system through a range of advance ratios from 0 to 0.14 along a $60^{\circ}$ sideslip trajectory to starboard. The geometry of the rotor system, along with the range of advance ratios that were studied, was chosen to be similar to those used during the HELIFLOW experiments in order that qualitative comparisons with the data from that project can be made. There are insufficient data available within the public domain, however, to allow an exact representation of the MR-TR system that was tested during the HELIFLOW experiments. Unfortunately, therefore, rigorous quantitative comparisons between the predictions made using the VTM and the HELIFLOW measurements cannot be made. The main rotor was controlled to ensure both zero longitudinal and lateral disk tilt and a constant thrust coefficient of 0.0068 , which is typical for the main rotor of a medium-sized helicopter in low-speed flight. Importantly, the tail rotor blades were maintained at a constant collective pitch of $10^{\circ}$ throughout all of the simulations that are presented in this paper; therefore, the overall yaw equilibrium of the helicopter was not satisfied at each of the flight conditions simulated. While not representative of practical flight, where the pilot would adjust the tail rotor collective pitch to obtain the desired torque, this simplification is important, as it allows the influence of MR-TR aerodynamic interaction on the performance of the tail rotor to be exposed without contamination from the control response of the tail rotor.

Top-aft and top-forward tail rotor configurations were simulated in both of the two vertical locations with respect to the main rotors that were used during the HELIFLOW experiments, as illustrated in

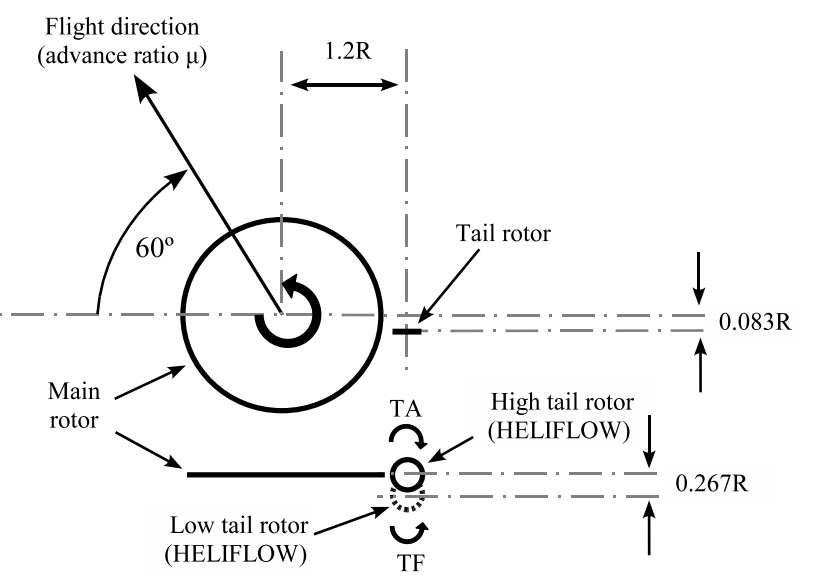

Fig. 2 Schematic showing the plan view and side view of the simulated MR-TR system.

Fig. 2. For clarity, the terms "low tail rotor" and "high tail rotor" are used throughout this paper to refer to the two specific positions in which the tail rotor was located during the HELIFLOW project, which are defined in Fig. 2. In addition, the tail rotor was simulated in four vertical locations that did not feature within the HELIFLOW experiments but complete a set of MR-TR configurations in which the location of the tail rotor was varied in uniform increments from $0.089 R$ above to $0.356 R$ below the main rotor hub. The flap dynamics of the tail rotor blades were suppressed by using a deltathree (pitch-flap coupling) angle of $45^{\circ}$. A description of the main and tail rotors (as simulated using the VTM) is given in Table 1.

\section{Effect of Tail Rotor Sense of Rotation on Directional Control Authority}

The measurements that were made during the HELIFLOW experiments (and reproduced in Fig. 1) provide an indication of the region of the flight envelope in which the performance of the tail rotor is dependent on its sense of rotation and furthermore show that this region is dependent on the vertical location of the tail rotor. The inference that may be made from Fig. 1 by helicopter engineers is incomplete, however, because it is unclear as to whether the difference in the performance of the top-aft and top-forward tail rotors persists to higher flight speeds than those measured. Figures 3 and 4 show the variation in the thrust coefficient of the VTM-simulated tail rotor over a range of flight speeds from hover to a helicopter advance ratio of 0.14 . In each figure, the thrust coefficient that is developed by tail rotors that are configured with either a top-aft or a top-forward sense of rotation are contrasted with the thrust coefficient that was developed by an equivalent tail rotor that was operated in isolation from the main rotor. Figure 3 shows the variation with advance ratio of the thrust coefficient of the low tail rotor, while Fig. 4 shows the equivalent variation in the thrust coefficient of the high tail rotor. The selection of the MR-TR configurations that are represented in Figs. 3 and 4 allow the VTM-predicted performance of the tail rotor to be

Table 1 Rotor data

\begin{tabular}{lcc}
\hline \hline & Main rotor & Tail rotor \\
\hline Number of blades & 4 & 4 \\
Rotor radius & $R$ & $R_{t}=0.167 R$ \\
Blade chord & $0.08 R$ & $0.2 R_{t}$ \\
Solidity & 0.086 & 0.186 \\
Twist & $-8^{\circ}$ (linear) & $0^{\circ}$ \\
Airfoil & RAE 9646 & NACA 0012 \\
Rotational speed & $\Omega$ & $\Omega_{t}=6.03 \Omega$ \\
Sense of rotation & counterclockwise & top-aft or \\
& from above & top-forward \\
\hline \hline
\end{tabular}

${ }^{\mathrm{a} R A E}$ denotes Royal Aircraft Establishment. 


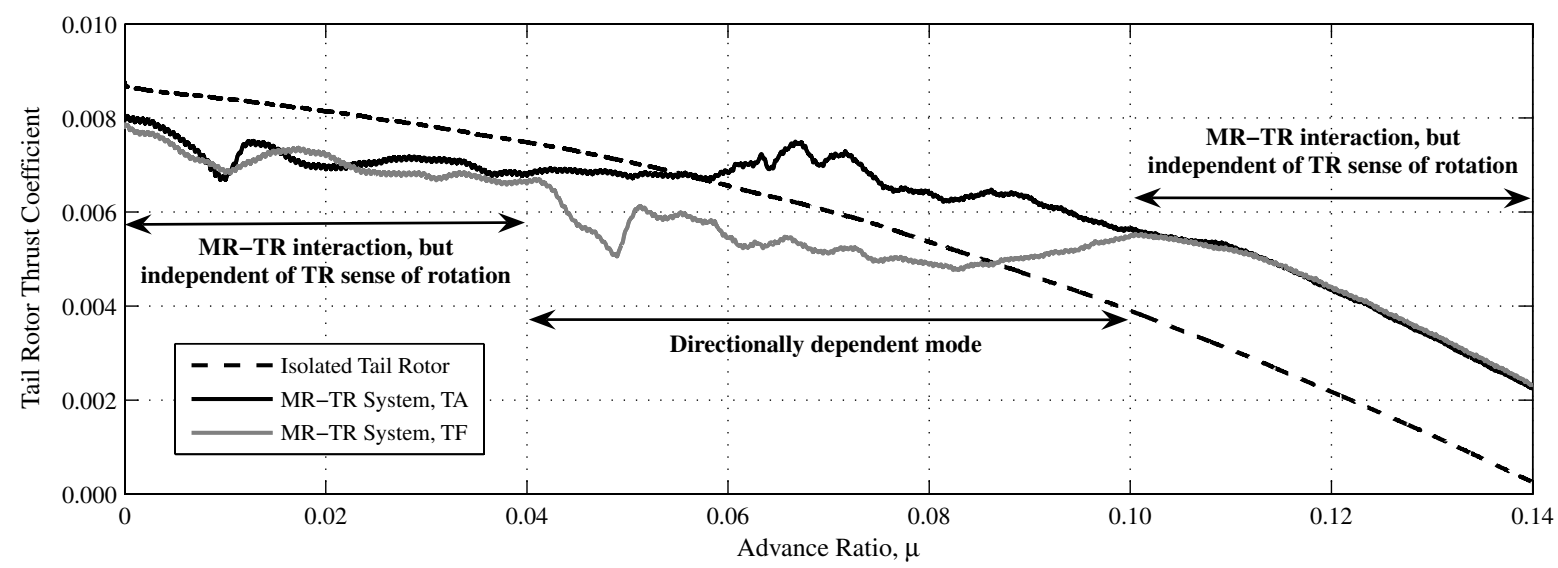

Fig. 3 Variation in the thrust coefficient developed by the low tail rotor as the MR-TR system accelerates gradually along a $60^{\circ}$ sideslip trajectory (TA: top-aft tail rotor, TF: top-forward tail rotor).

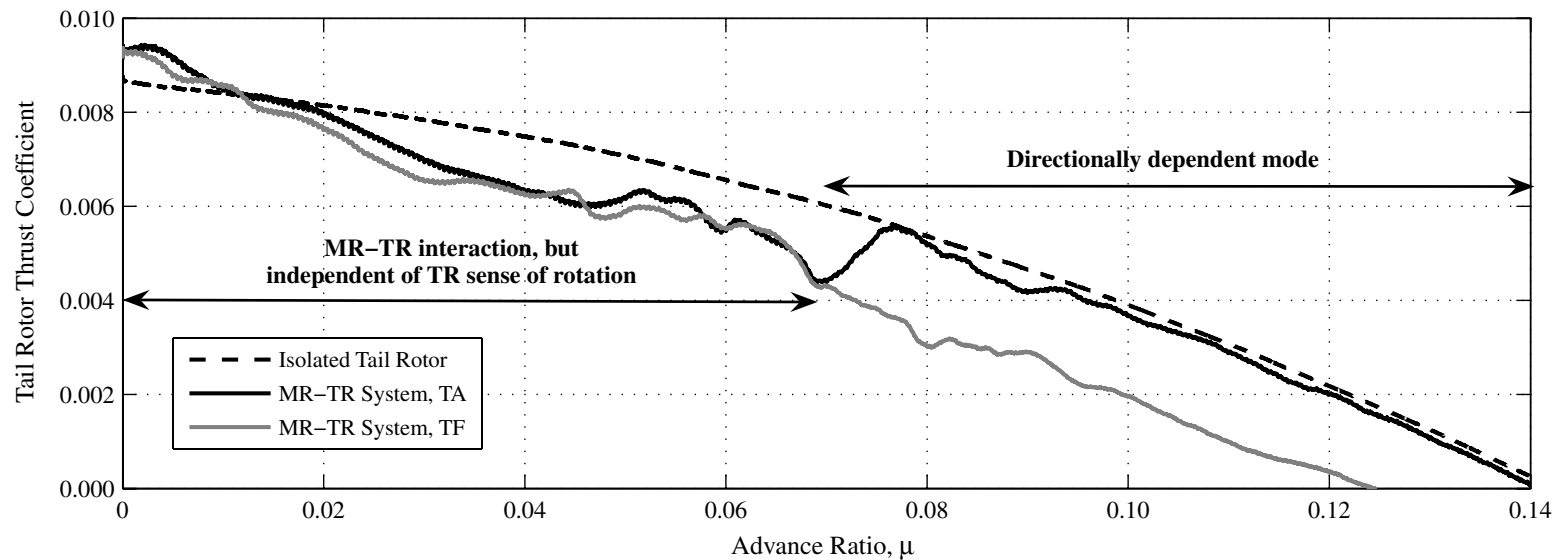

Fig. 4 Variation in the thrust coefficient developed by the high tail rotor as the MR-TR system accelerates gradually along a $60^{\circ}$ sideslip trajectory (TA, top-aft tail rotor; TF, top-forward tail rotor).

related, to some extent, to the thrust measurements that were made during the HELIFLOW experiments.

The data that are shown in Figs. $\underline{3}$ and $\underline{4}$ (and Fig. $\underline{5}$ later in this paper) were computed using one single simulation for each different MR-TR configuration, during which the advance ratio of the system was slowly increased throughout. This method of simulating the operation of the MR-TR system was necessary to ensure that resolving the variation in the performance of the tail rotor with flight speed over the necessary range of advance ratios was computationally tractable. Importantly, the rate at which the MR-TR system was accelerated was low compared with the rate at which the rotor aerodynamics and wake structure evolved. To clarify the underlying variation of the tail rotor thrust coefficient over the full range of simulated advance ratios, the data that are shown in Figs. 3 and 4 (and Fig. 5) have been filtered by removing any oscillations at frequencies equal to or above that of main rotor blade passage. The careful application of this filter exposes the characteristic variability in the tail rotor thrust coefficient at frequencies below that of main rotor blade passage, without erroneously modifying either the amplitude or the remaining frequency components of the tail rotor loading. The direction of rotation of the isolated tail rotor is irrelevant in Figs. $\underline{3}$ and 4 , because the thrust coefficient of the tail rotor is independent of the sense of rotation of the rotor, provided that it operates in isolation from the main rotor. Finally, the performance of the VTM-simulated tail rotor that is shown in Figs. 3 and 4 is presented directly in terms of the rotor thrust coefficient, rather than by using the scaled form of rotor thrust (labeled "tail rotor thrust ratio" in Fig. 1) in which the HELIFLOW data were published [8]. It is the opinion of the authors that scaling using the thrust attained in hover is misleading, as it implies that some form of generalization of the tail rotor performance has been achieved. If the tail rotor thrust is scaled in this way, it will still depend on the specific value of the tail rotor thrust in hover as the advance ratio of the system is varied; thus, the scaling merely obscures comparison of the performance of tail rotors with differing configurations.

Figures $\underline{3}$ and $\underline{4}$ demonstrate that, at a very low advance ratio, the VTM-simulated tail rotors with top-aft or top-forward senses of rotation develop a very similar thrust coefficient for the same collective pitch setting, but this differs, to some extent, from the thrust coefficient of the tail rotor when it is operated in isolation. As such, although the performance of the tail rotor at a very low advance ratio is influenced by its interaction with the main rotor, the thrust coefficient that the tail rotor develops remains essentially independent of its sense of rotation. The MR-TR system differs from the isolated tail rotor only by the addition of the main rotor, while the other geometric and trim characteristics of the two systems are identical. This implies that the thrust that is developed by the tail rotor when the combined MR-TR system is operated at very low advance ratio is directly influenced by the wake that is produced by the main rotor. Indeed, previous work by Balch [17] and Fletcher and Brown [18] has shown that there is significant aerodynamic coupling between the main and tail rotors of a conventional helicopter in hover and in low-speed flight. A comparison of Figs. $1, \underline{3}$, and $\underline{4}$ shows that the mode of operation in which the VTM-predicted thrust coefficient of the tail rotor is insensitive to its direction of rotation is consistent with the measurements that were made during the HELIFLOW project when the system was operated at similarly low advance ratios.

If the MR-TR system translates at a moderately higher advance ratio, the aerodynamic performance of the tail rotor is characterized by a second, or "directionally dependent," mode, in which the thrust 
coefficient of the rotor is governed significantly by its sense of rotation. The VTM simulations show that, while operating within the directionally dependent mode, the tail rotor with the top-aft sense of rotation develops a significantly greater thrust coefficient than the equivalent tail rotor with the top-forward sense of rotation. Both the experimental measurements that are shown in Fig. 1, and the VTM simulations that are shown in Figs. 3 and $\underline{4}$, illustrate that the tail

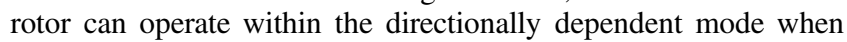
positioned at either of the two vertical locations that are represented in these figures. The advance ratio at which the onset of the directionally dependent mode occurs varies appreciably, however, with the vertical location of the tail rotor and, for practical helicopter geometries, the onset of this mode is likely to be at advance ratios in the range 0.04-0.08. Both the HELIFLOW measurements and the VTM predictions illustrate that the advance ratio at which the thrust coefficient of the top-aft tail rotor is largest when compared with the top-forward tail rotor will also vary considerably with the vertical location of the tail rotor.

Only a limited set of tail rotor thrust measurements are available from the HELIFLOW tests at advance ratios above 0.08 . Figure $\underline{1}$ does show, however, that at the upper limit of those advance ratios that were tested, there is a significant reduction in the difference between the thrust coefficient produced by the low tail rotor with the top-aft sense of rotation and the thrust coefficient of the same rotor when configured to rotate top-forward. This trend suggests that the sensitivity of the performance of the tail rotor to its direction of rotation may reduce significantly at flight speeds higher than those for which measurements were taken during the HELIFLOW project. In the past, little attention has been afforded to the performance of the tail rotor when the helicopter operates in sideslip at advance ratios above 0.1 . Indeed, before the current work, there were insufficient data available within the open literature to show conclusively how the sense of rotation of the tail rotor determines its thrust coefficient at high helicopter advance ratios. The VTM simulations of the low tail rotor configuration that are shown in Fig. 3 demonstrate clearly, however, that the rotor operates within the directionally dependent mode for only a finite range of advance ratios. In contrast, Fig. 4 shows that the performance of the high tail rotor remains highly dependent on its sense of rotation, even at relatively high advance ratios in the range $0.12-0.14$. The data presented in Figs. $\underline{3}$ and $\underline{4}$ suggest, therefore, that the vertical location of the tail rotor governs, to some extent, whether or not the directionally dependent mode is bound to a finite range of advance ratios at which the helicopter may operate.

The two distinct modes of tail rotor performance that are indicated in Figs. 3 and 4 highlight the variability with flight speed of the interaction between the main and tail rotors, and they illustrate clearly the need to better understand the aerodynamic mechanism by which this interaction influences the tail rotor loading. It is important, however, to first place both the VTM simulations and the HELIFLOW measurements within context. During flight at relatively high speed and at a high angle of sideslip to starboard, the tail rotor modeled in the present simulations will operate at a significant effective rate of ascent. Indeed, in each of the MR-TR configurations that are represented in Figs. 3 and 4, the thrust coefficient of the tail rotor reduces as the advance ratio of the helicopter is increased. This behavior is expected, given that the collective pitch of the tail rotor blades was held constant, and it can be considered as the underlying trend onto which the influence of the MR-TR interaction is superimposed. It is important to note, however, that the reduction in thrust coefficient of the tail rotor with advance ratio is forced, to some extent, by the fact that the collective pitch of the blades was held constant in both the VTM simulations and the HELIFLOW experiments. This mode of operation is not representative of practical flight, as the collective pitch of the tail rotor blades would be varied to enable the helicopter to be trimmed properly in yaw. As such, any degradation of directional control authority would be measured by how much the available pedal travel, and hence the available collective pitch, is reduced after the helicopter is trimmed in yaw at a particular flight configuration. In practice, crosswind flight at high angles of sideslip would be inhibited at advance ratios above those considered here, because the directional control of the aircraft would be limited by either the stall of the tail rotor or by excessive pedal travel.

\section{Influence of Tail Rotor Vertical Location}

Figure 5 shows the VTM-simulated thrust coefficient that is developed by tail rotors with top-aft and top-forward senses of rotation as the advance ratio of the system varies from 0.00 to 0.14 but with the tail rotor situated in six different vertical locations. The thrust coefficient that is developed by both the top-aft and the topforward tail rotors is shown in Fig. $\underline{5}$, alongside the thrust coefficient that is produced by the tail rotor when operating in isolation. The thrust coefficient data that are shown in Fig. 5a were obtained by simulating the tail rotor at the highest of the set of six prescribed tail rotor locations ( $0.089 R$ above the main rotor hub). In the sequence of Figs. $5 \mathrm{~b}-5 \mathrm{f}$, the tail rotor was lowered by increments of $0.089 R$, so that the thrust coefficient data that are shown in Fig. $5 \mathrm{f}$ were obtained by simulating the tail rotor in the lowest of the set of six positions ( $0.356 R$ below the main rotor hub). The high and the low tail rotors that are specifically referred to elsewhere in this paper are represented in Figs. $5 \mathrm{~b}$ and $5 \mathrm{e}$, respectively. Figure 6 illustrates the variation with the vertical location of the tail rotor of the range of advance ratios over which the tail rotor operates within the directionally dependent mode. Figure 6 also illustrates those regions of the flight envelope in which the tail rotor may interact with the main rotor wake but where the performance of the tail rotor is not influenced by its sense of rotation. The data that are presented in Fig. $\underline{6}$ were extracted by identifying the advance ratio at which the thrust coefficients of the top-aft and top-forward tail rotors first started to differ significantly and persistently.

At its highest position, the interaction between the main and tail rotors leads to both the top-aft and the top-forward tail rotors developing a slightly larger thrust coefficient than the isolated tail rotor when operating at advance ratios just beyond hover, as shown in Fig. 5a. Lowering the tail rotor with respect to the main rotor has the effect of degrading the performance of the tail rotor in very low-speed flight compared with the performance of the isolated tail rotor. As an indication of this variation with tail rotor position, Fig. 5a shows that when the tail rotor is operated at an advance ratio of 0.02 and is located at the highest of the six vertical locations that were simulated, it develops a thrust coefficient that is approximately equal to that of the isolated tail rotor. In contrast, Fig. 5f shows that when the tail rotor is simulated in the lowest of the $\bar{M} R-T R$ configurations and operated at an advance ratio of 0.02 , it develops a thrust coefficient that is approximately $15 \%$ smaller than that of the isolated tail rotor. It should be noted that this observation is, to some extent, at odds with the HELIFLOW measurements that are shown in Fig. 1. The HELIFLOW data can be interpreted as implying little sensitivity to the vertical position of the tail rotor when the MR-TR system operates in very low-speed flight. Given the uncertainty regarding some aspects of the setup of the HELIFLOW model, these discrepancies may correspond simply with a difference in the thrust coefficient and disk tilt of the main rotor, or in the collective pitch of the tail rotor, between the HELIFLOW experiment and the present numerical simulations. It does highlight, however, where a need may exist for a more extensive set of experimental measurements to better understand the effects of MR-TR interaction on the performance of the tail rotor in very low-speed flight.

Figure 6 suggests that there is a gradual, but somewhat nonlinear, increase with the vertical location of the tail rotor of the advance ratio at which the rotor enters the directionally dependent mode. Figures $5 d-5 f$ show clearly that when the tail rotor is located in a relatively low position, an advance ratio exists beyond which the tail rotor ceases to operate within the directionally dependent mode, and the thrust coefficient of the top-aft tail rotor returns to being approximately equal to that of the top-forward tail rotor. Indeed, Fig. 6 indicates that there is a precipitous increase in the advance ratio at which the directionally dependent mode terminates as the vertical location of the tail rotor is increased. In the simulations in which the tail rotor was located in a relatively high position, shown in 


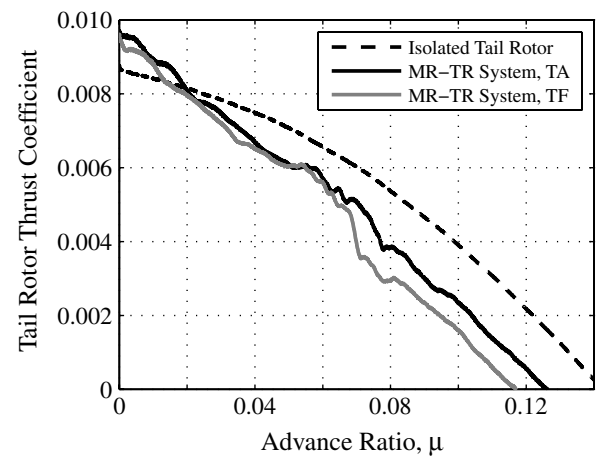

a) $h_{t}=0.089 R$

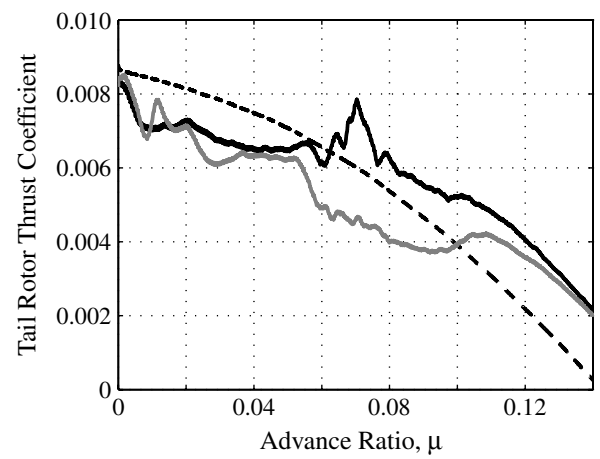

d) $h_{t}=-0.178 R$

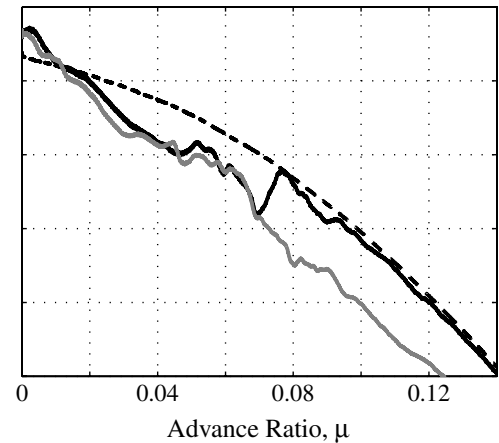

b) $h_{t}=0 R$ (high tail rotor)

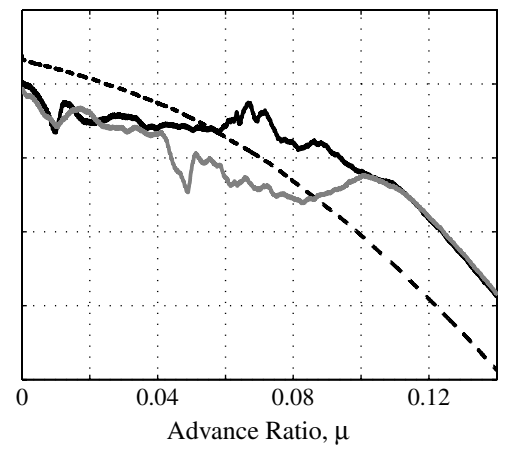

e) $h_{t}=-0.267 R$ (low tail rotor)

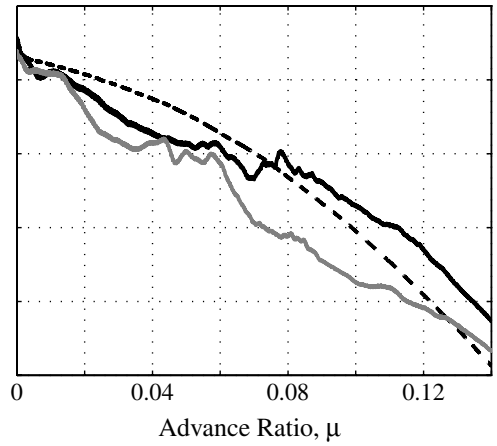

c) $h_{t}=-0.089 R$

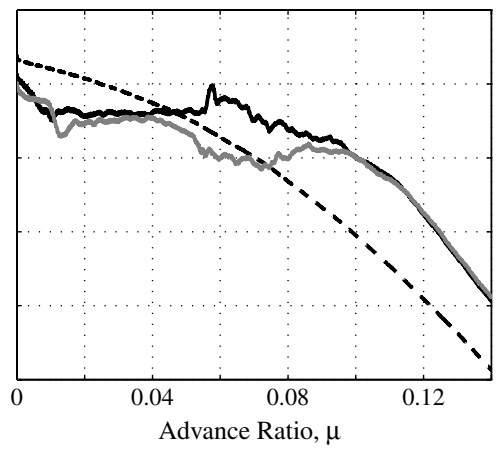

f) $h_{t}=-0.356 R$

Fig. 5 Dependence of tail rotor thrust coefficient, as a function of helicopter advance ratio, on the vertical location of the tail rotor with respect to the main rotor (TA: top-aft tail rotor, TF: top-forward tail rotor). Note that when $h_{t}=0 R$, the tail rotor hub is at the same height as the main rotor hub.

Figs. 5a-5c, the thrust coefficients of the rotors with opposing senses of rotation remain significantly different, even at the highest advance ratios that were simulated. While it is possible that even those tail rotors that are located highest with respect to the main rotor may exit from the directionally dependent mode at advance ratios greater than those simulated here, the thrust coefficient of the tail rotor would reduce to zero if the collective pitch were to be held constant. As such, these data would yield little further insight into the aerodynamic mechanism that causes the directionally dependent mode. Figure 6 clearly illustrates that as the tail rotor is lowered with respect to the main rotor, the range of advance ratios over which the thrust coefficient of the tail rotor is directionally dependent is reduced. Furthermore, Fig. 6 suggests that the size of the difference between the thrust coefficients of the top-aft and top-forward tail rotors is determined, to some extent, by the relative positions of the main and tail rotors.

The directionally dependent mode is created by the immersion of the tail rotor within the main rotor wake and the influence that the associated rotational flow has on the local dynamic pressure at the tail rotor blades. For identical control settings, top-aft tail rotors develop more thrust than those with the opposing top-forward configuration, because the flowfield that is induced by the main rotor results, to some extent, in an increase in local dynamic pressure at the tail rotor. The variability in the advance ratios that mark the onset and end of the directionally dependent mode, shown in Fig. $\underline{6}$, can be understood by considering the effect that the vertical location of the tail rotor has on its position within the main rotor wake. Figure 7 shows a series of instantaneous snapshots of the wake that is induced by the main and tail rotors when the system is operating in steady flight at the three different advance ratios of $0.02,0.07$, and 0.12 . Those snapshots on the top row of Fig. 7 are of the wake of the MR-TR system with the low tail rotor, while the snapshots on the bottom row of the figure are of the wake of the MR-TR system with the high tail rotor. The flight speeds that are represented in Fig. 7 were selected because both the low and the high tail rotors demonstrated distinct differences in performance compared with that of the isolated tail rotor, as shown in Figs. 3 and $\underline{4}$, respectively. In each of the simulations that are shown in Fig. 7, the tail rotor has a top-forward sense of rotation. The geometry of the wakes of the main rotor (light gray) and the tail rotor (dark gray) are represented in Fig. 7 by plotting surfaces on which the vorticity in the flow around the rotors is constant. The specific isosurfaces of vorticity that are shown in Fig. 7 have been chosen to emphasize the spatial extent of the induced flow rather than finerscale structures, such as individual vortex filaments.

Figures $7 \mathrm{a}$ and $7 \mathrm{~d}$ show that, when the MR-TR system operates at a relatively low advance ratio, the wake that is induced by the main rotor is skewed modestly, but it otherwise strongly resembles the tubular wake that is developed by a hovering rotor. It should be noted that the wake that is developed by a main rotor is known to transform at moderate advance ratios from the tubular structure that is induced by a hovering rotor into a more planar structure, in which the wake

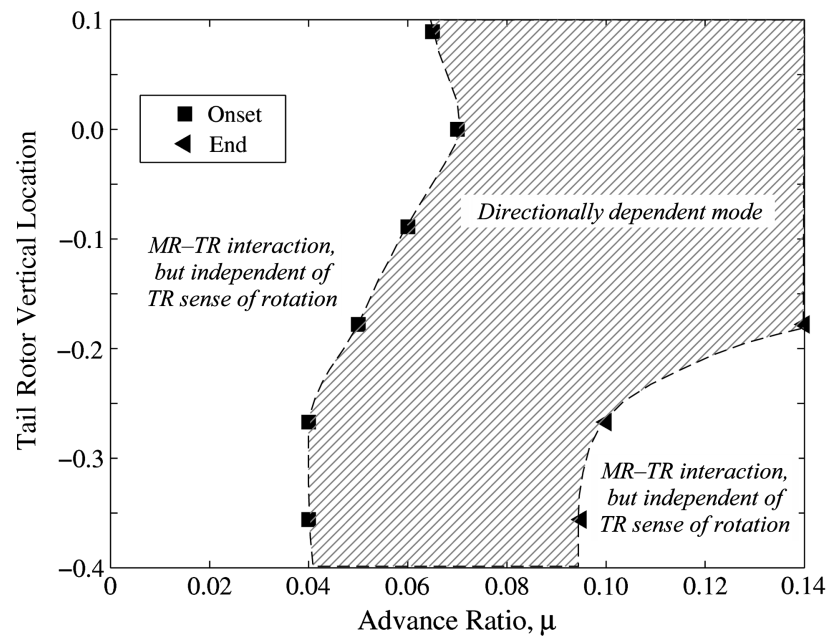

Fig. 6 Variation with vertical location of the range of advance ratios over which the tail rotor operates within the directionally dependent mode. 


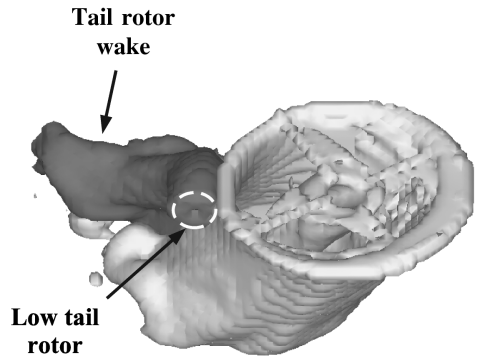

a)

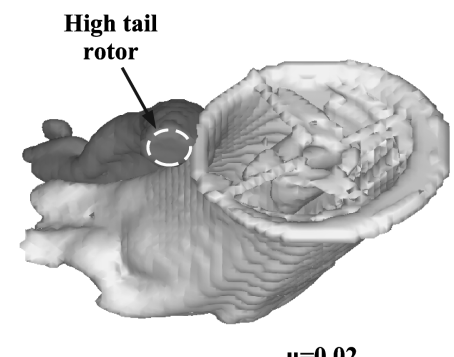

d)

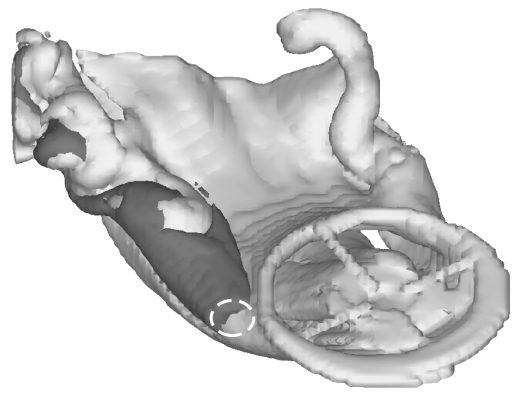

b)

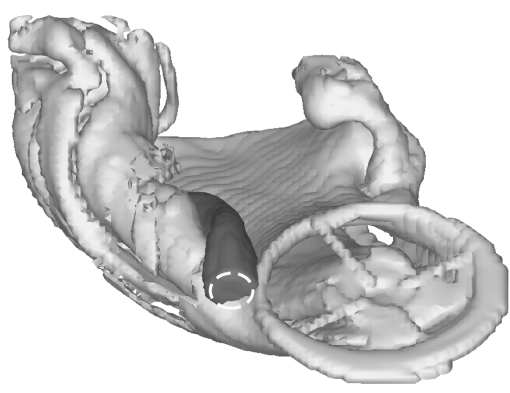

$\boldsymbol{\mu}=\mathbf{0 . 0 7}$

e)

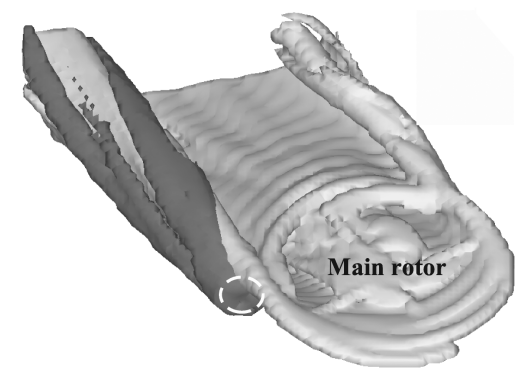

c)

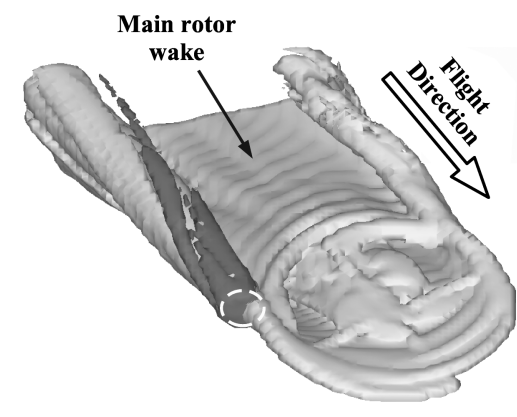

$\boldsymbol{\mu}=\mathbf{0 . 1 2}$

f)

Fig. 7 Instantaneous snapshots of the main rotor and tail rotor wakes that represent the interaction between the rotors at three pertinent advance ratios. MR-TR systems with low and high tail rotors are shown at the top and bottom, respectively.

rolls up toward the edges (similar to the wake trailed behind a fixedwing aircraft while cruising). This transformation in the morphology of the main rotor wake generally occurs at an advance ratio of approximately 0.1 . This advance ratio does not vary significantly with either the configuration or the trim conditions of the helicopter. A helicopter operating at a posttransitional advance ratio of 0.12 would be expected, therefore, to develop a more structured, periodic wake than the same helicopter operating at the lower, pretransitional, advance ratio of 0.07 . Though Figs. $\underline{3}$ and $\underline{4}$ indicate that the tail rotor does interact aerodynamically with the main rotor when the MR-TR system translates at very low advance ratio, Figs. 7a and 7d suggest that the tail rotor is not impinged upon significantly by the main rotor wake, notwithstanding whether it is mounted in either the low or the high positions shown. When the MR-TR system operates at a moderately higher, but still pretransitional, advance ratio, the concentrated vortices that are trailed from the tips of the main rotor blades cluster at the lateral extremities of the wake as they convect downstream from the rotor disk. Figure $7 \mathrm{~b}$ illustrates that when the MR-TR system with the low tail rotor operates at an advance ratio of 0.07 , this cluster of vortices impinges on a significant portion of the tail rotor. In contrast, Fig. 7e shows that, at the same advance ratio, only a very small portion of the high tail rotor is impinged upon by the main rotor wake. Figure $7 \mathrm{e}$ suggests, therefore, that the thrust coefficient of the high tail rotor is largely insensitive to its direction of rotation at advance ratios of less than 0.07 , because the tail rotor does not interact sufficiently with the region of the main rotor wake where there is significant rotational flow.

Both the low and the high tail rotors are partially immersed within the main rotor wake when the MR-TR system operates at posttransitional advance ratios. Figure $7 \mathrm{c}$ shows that the main rotor wake impinges on only a small portion of the upper half of the low tail rotor, while Fig. $7 \mathrm{f}$ illustrates that the wake impinges significantly on the lower forward quadrant of the high tail rotor. A comparison of Figs. 3 and $\underline{4}$ shows that when the MR-TR system translates at an advance ratio of 0.12 , the thrust coefficient that is developed by the low tail rotor is insensitive to its direction of rotation, whereas the high tail rotor operates deep within the directionally dependent mode, and a significant difference between the thrust coefficients of the top-aft and top-forward tail rotors exists. A comparison of Figs. $\underline{7 c}$ and $\underline{7 f}$ suggests that this difference in the operational mode of the tail rotor is, to some extent, caused simply by the position of the tail rotor with respect to the main rotor wake. Indeed, the vertical location of the tail rotor with respect to the main rotor is shown by Fig. $\underline{6}$ to influence significantly the range of advance ratios at which a given tail rotor will operate within the directionally dependent mode. Thus, it can be surmised in relatively simple terms that, if the tail rotor is located in a relatively high position, the main rotor wake would need to skew further aft in order for it to impinge on the tail rotor, and this would delay the onset of the directionally dependent mode to higher advance ratio.

\section{Aerodynamic Origin of the Directionally Dependent Mode}

When the MR-TR system is flown along the trajectories that have been simulated in this paper, the thrust coefficient that is developed by the tail rotor, when operated in isolation from the main rotor, is insensitive to the tail rotor's direction of rotation. It can be deduced, therefore, that the directionally dependent mode must be caused by the interaction between the tail rotor and the main rotor or, more specifically, the wake that is induced by the main rotor. Indeed, the significance of the interaction between the main and tail rotors is shown by the variation with the position of the tail rotor of the range of advance ratios over which the directionally dependent mode exists. In this section of the paper, the relationship between the thrust that the tail rotor develops and the vorticity within the main rotor wake will be examined. Notwithstanding the transition in the morphology of the main rotor wake at moderate advance ratios, the sense of rotation of the tail rotor has been shown to strongly influence its performance when the MR-TR system operates at either pretransitional or posttransitional advance ratios, providing that the position of the tail rotor is such that it is immersed sufficiently within the main rotor wake. To better understand the variation in the directionally dependent mode as the morphology of the main rotor wake transitions, two specific combinations of MR-TR geometry and advance ratio will now be examined: the low tail rotor system when operating at the steady (pretransitional) advance ratio of 0.07 and the high tail rotor system when translating at the posttransitional advance ratio of 0.12 . Using MR-TR systems with different tail rotor positions does complicate the analysis slightly, but it is essential in 


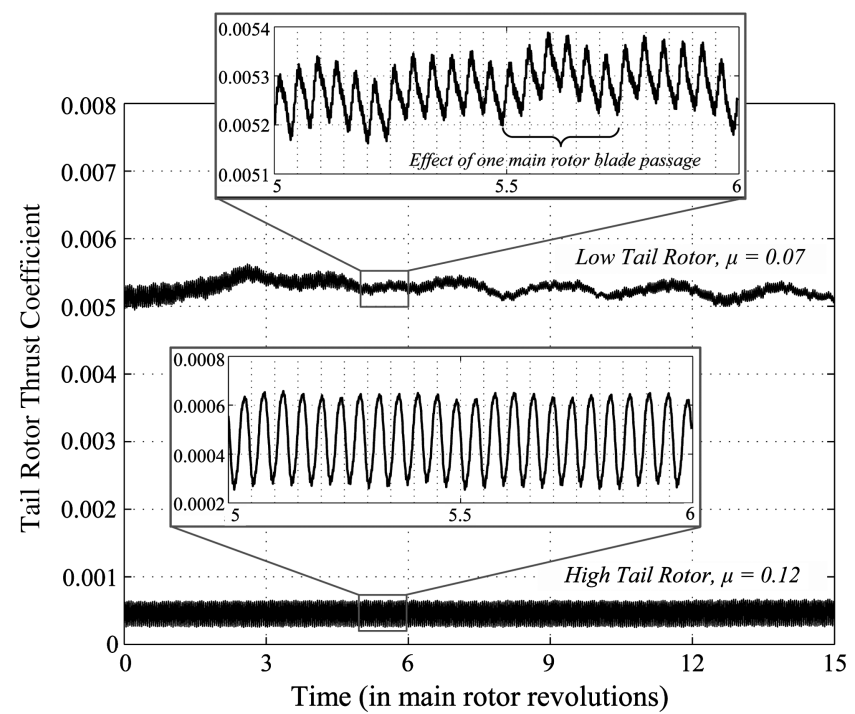

Fig. 8 Thrust coefficient developed by the low tail rotor at an advance ratio of 0.07 and the high tail rotor at an advance ratio of 0.12 . In both cases, the tail rotor has a top-forward sense of rotation.

order that the aerodynamic mechanism that causes the directionally dependent mode can be exposed when the rotor system operates at both pretransitional and posttransitional advance ratios.

Figure 8 demonstrates the temporal variability of the thrust coefficient that is developed by the tail rotor when it is simulated in each of the two configurations described previously. In both of the simulations that are shown in Fig. 8, the tail rotor was configured to rotate in the top-forward sense. Comparable unsteadiness was also observed, however, in the thrust coefficient of the top-aft tail rotor when it was simulated at the same flight conditions as those represented in Fig. $\underline{8}$. It should be noted that Fig. $\underline{8}$ shows only the aerodynamic forcing that is developed on the tail rotor, and it does not represent the forcing that would be transmitted to the rotor shaft, which would thus be modulated by the dynamics of the blades. Figure 8 shows that when the low tail rotor is operated at a pretransitional advance ratio, the temporal variability in its thrust coefficient is composed of three principal constituents: fluctuations at the blade passage frequency of the tail rotor (approximately 24 per main rotor revolution), modulation at the blade passage frequency of the main rotor (four per main rotor revolution), and an aperiodic meandering over a time scale that is significantly longer than the period of main rotor revolution. In contrast, Fig. $\underline{8}$ demonstrates that when the high tail rotor is operated at an advance ratio of 0.12 (i.e., beyond transition), the temporal variability of its thrust coefficient is composed almost entirely of fluctuations at the blade passage frequency of the tail rotor, with only negligible fluctuations at lower frequencies. In addition, the fluctuations in the thrust coefficient at the blade passage frequency of the tail rotor are of higher amplitude when the MR-TR system operates at a posttransitional rather than a pretransitional advance ratio.

The presence of fluctuations in the thrust coefficient of the low tail rotor at the blade passage frequency of the main rotor indicates that the tail rotor interacts, to some extent, with the individual tip vortices that are trailed downstream of the main rotor. When the MR-TR system operates within the pretransitional regime, the main rotor wake exhibits significant aperiodic meandering. This meandering causes the aperiodic variation in the thrust coefficient of the low tail rotor that is shown in Fig. . The freestream velocity of the MR-TR system leads the tail rotor to develop an asymmetry in aerodynamic loading, in which the blades develop a higher loading when they traverse the advancing side of the disk than when they traverse the retreating side. This asymmetry in blade loading will result in oscillations in the thrust coefficient of the tail rotor at its blade passage frequency, and these oscillations would occur even if the rotor were to be operated in isolation. The amplitude of the inherent oscillations in thrust that are produced by both the low and the high tail rotors is, however, relatively small. The oscillations in the thrust coefficient of the high tail rotor (shown in Fig. 8) are, however, significantly larger in amplitude than would be experienced by the same rotor if it were operated in isolation. Figure 8 suggests, therefore, that the impingement of the main rotor wake on the tail rotor causes additional variability in the aerodynamic loading on the blades that amplifies the unsteadiness within the thrust coefficient that the tail rotor produces.

To analyze the origin of the unsteadiness in the tail rotor thrust coefficient that is shown in Fig. 8, it is necessary to simulate the MRTR system to a high enough resolution that the finer-scale vortical structures can be characterized. This requires a computational mesh with a cell density that is significantly higher than that used to compute the snapshots of the wake that are shown in Fig. 7. To achieve this, however, the aerodynamic discretization of the blades should also be increased so that the distribution of vorticity that is trailed and shed from the blades is also resolved at equivalently small scales. To demonstrate the consistency of the VTM simulations, Fig. 9 shows a comparison of the radial distributions of the blade loading coefficient on the main rotor blades that were predicted using two different forms of discretization. In the low-resolution

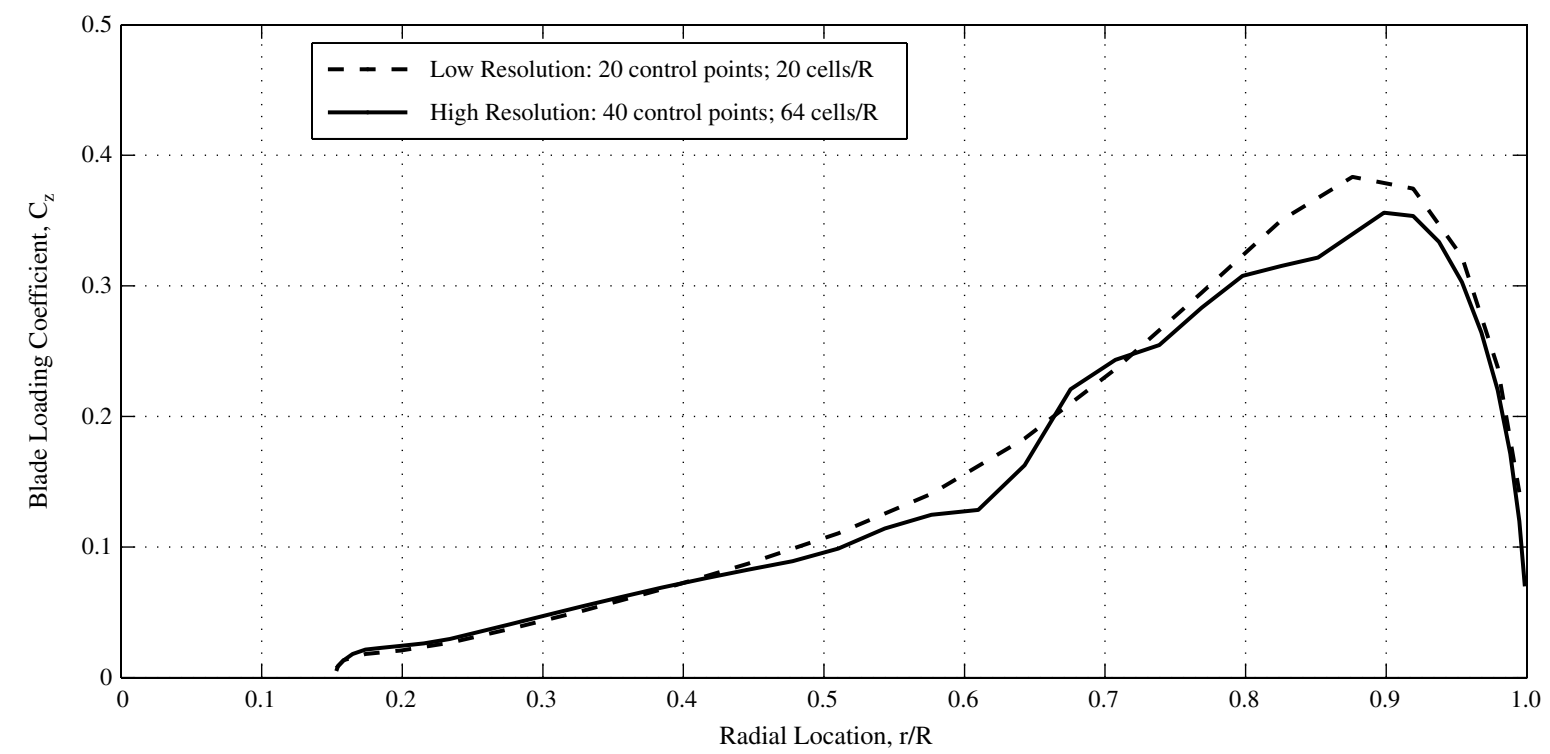

Fig. 9 Comparison of the loading on the main rotor blades when at an azimuth of $90^{\circ}$, computed using two different combinations of blade discretization and grid cell density. The tail rotor is configured in the low position with a top-forward sense of rotation. Advance ratio $=0.07$. 
simulation that is shown in Fig. 9 , the aerodynamics of the main rotor blades were modeled using $2 \overline{0}$ control points, and the MR-TR system was immersed within a computational grid with a cell density of 20 cells per main rotor radius. The high-resolution simulation was performed using 40 control points along the length of the main rotor blades and a grid with a cell density of 64 cells per main rotor radius. Figure 9 shows that the distribution of loading on the main rotor blades is entirely consistent, but that some additional small perturbations to the underlying loading distribution are predicted by the high-resolution simulation. These perturbations are caused by localized fluctuations within the flow velocity at the blades as they interact with the tip vortices that propagate aft across the main rotor. These blade-vortex interactions are not well resolved within the lowresolution simulation because of the more diffuse, coarser representation of individual vortex filaments within the computational domain and the subsequently less impulsive fluctuations that they induce within the velocity field.

The temporal fluctuations in the thrust coefficient of the tail rotor that are shown in Fig. 8 suggest that the character of the interaction between the tail rotor and the main rotor wake when the MR-TR system translates at pretransitional advance ratios is considerably different than the character of the interaction at posttransitional advance ratios. At low speed, the performance of the top-forward tail rotor is degraded, at least in part, by its interaction with individual main rotor tip vortices. In contrast, when the MR-TR system translates at a moderately higher advance ratio, Fig. 8 suggests that the performance of the top-forward tail rotor is not degraded by the effect of individual main rotor tip vortices but, rather, by the impingement of a coherent vortical structure that is generated from within the main rotor wake. As such, the suggestion that was made by Ellin [7] and others, specifically that the behavior of the tail rotor is governed by its interaction with a coherent supervortex, appears to be true only for some MR-TR configurations and only within a finite portion of the flight envelope of the aircraft.

To emphasize this conclusion, Fig. 10 shows the overall structure of the wake that is developed by the $\overline{\mathrm{MR}}$-TR system with a low tail rotor when translating at a pretransitional advance ratio simulated using the finer of the two discretizations described previously. In Fig. 10, the geometry of the wakes of the main rotor (light gray) and the tail rotor (dark gray) are represented by plotting surfaces on which the vorticity is constant. Figure 10 shows that the vortices that are trailed downstream of the tips of the main rotor blades at this advance ratio do not form a coherent vortex structure in the space just upstream of the tail rotor. Instead, the main rotor wake is characterized by a cluster of largely separate vortex filaments. Figure 11 shows an instantaneous snapshot of the wake structure that is developed by the MR-TR system with a high tail rotor when it is operating at a posttransitional advance ratio. In contrast to the main rotor wake that is shown in Fig. 10, Fig. 11 shows that, in the posttransitional flight regime, the individual vortices that are created behind the tips of the main rotor blades coalesce to form a coherent supervortex a very short distance downstream of the main rotor effectively before they reach the position of the tail rotor. Figure 12 shows the wake that is produced by the main rotor in the same two MR-TR configurations and flight conditions that are represented in Figs. 10 and 11 but, instead, the wake is rendered as a surface on which the vorticity is significantly higher, thus enabling the individual vortex filaments that are trailed behind the tips of the main rotor blades to be visualized more clearly. Only the portion of the main rotor wake that is closest to the tail rotor is shown in order to clarify the presentation. Figure 12 a shows clearly that when the MRTR system operates at a pretransitional advance ratio, the concentrated vortices that are trailed behind the blades, although significantly distorted by their mutual interaction, remain distinct up to the point at which they impinge on the tail rotor. In contrast, Fig. 12b shows that the tip vortices that are developed downstream of the main rotor when it operates at an advance ratio of $0.12 \mathrm{do}$, as suggested by previous researchers, coalesce to form a single vortex by the time that this rotational flow structure impinges on the tail rotor.

The insets to Fig. 10, labeled Figs. 10a-10d, represent the wake structure of the MR- $\overline{\mathrm{TR}}$ system in the space upstream of the tail rotor at intervals corresponding to $120^{\circ}$ of the main rotor azimuth, and they illustrate the fluctuations in the wake structure that occur over the time scale of one main rotor revolution. The fluctuations in the structure of the main rotor wake as it impinges on the tail rotor are aperiodic, but the data presented in Fig. 10 suggest that these fluctuations occur in a band of frequencies that are below that at which the main rotor rotates. These fluctuations are most likely to be responsible for the variation in tail rotor thrust at similarly low frequencies that was demonstrated in Fig. 8. Figures 11a-11d again show snapshots of the wake structure at intervals of $\overline{12} 0^{\circ}$ of main rotor azimuth, and they demonstrate the relative steadiness of the spatial distribution of vorticity within the main rotor wake in the

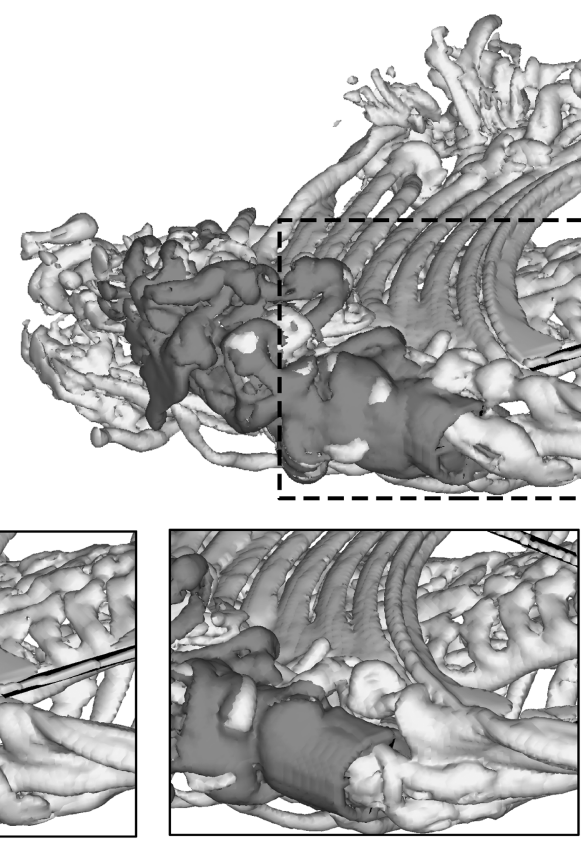

a) Main rotor azimuth (time), $\psi=0^{\circ}$ b) $\psi=120^{\circ}$

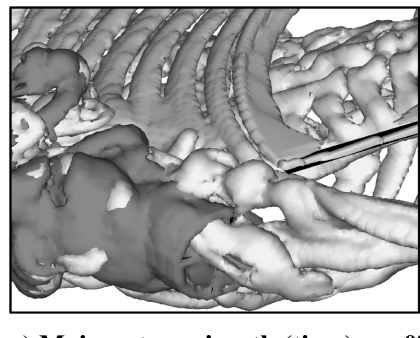

Fig. 10 Highly resolved instantaneous snapshot of the wake of a low tail rotor operating with the main rotor in $60^{\circ}$ sideslip at an advance ratio of 0.07 (light surface, main rotor wake; dark surface, tail rotor wake). 


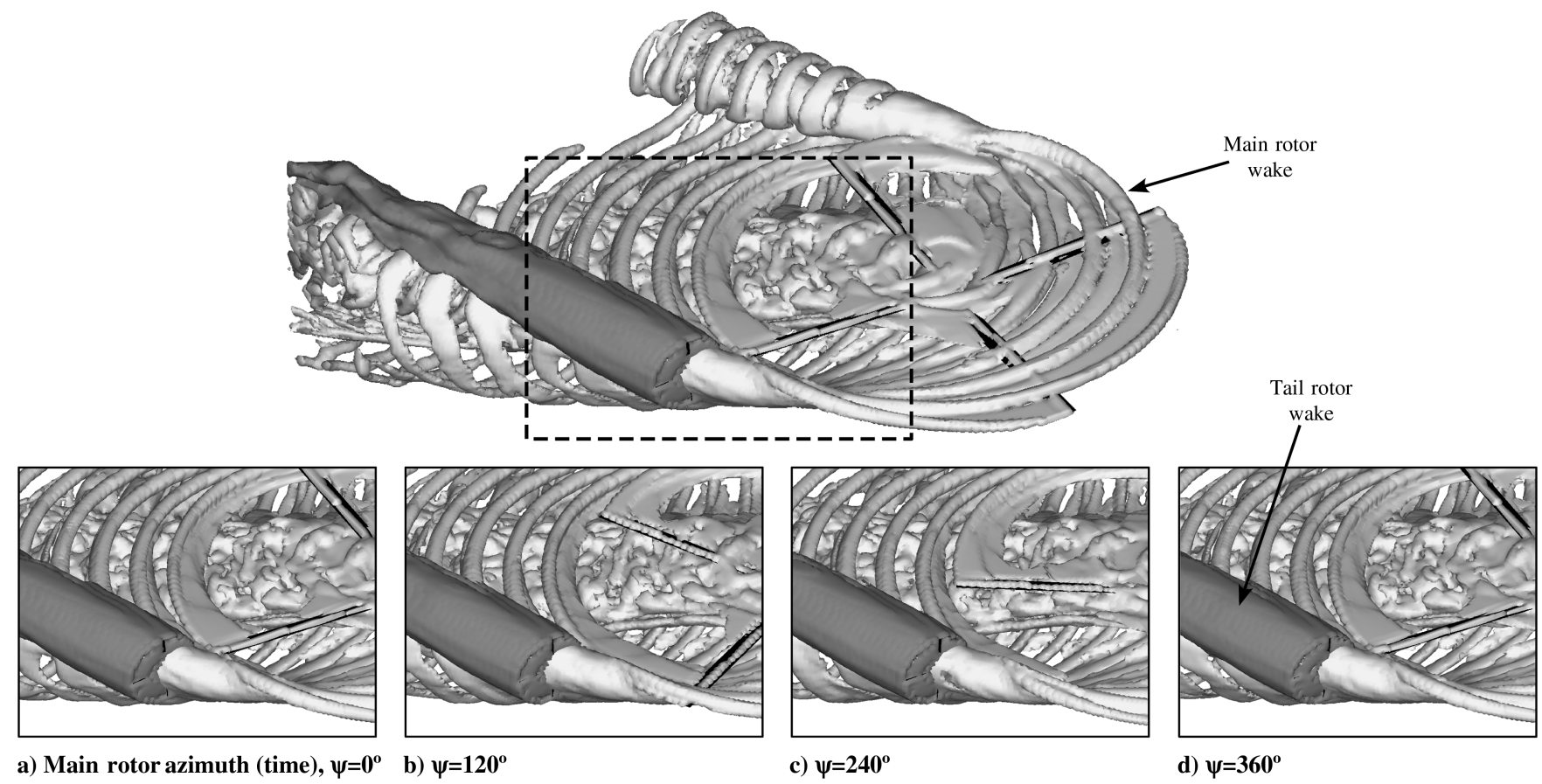

Fig. 11 Highly resolved instantaneous snapshot of the wake of a high tail rotor operating with the main rotor in $60^{\circ}$ sideslip at an advance ratio of 0.12 (light surface, main rotor wake; dark surface, tail rotor wake).

region ahead of the tail rotor when the system operates at a posttransitional advance ratio. Figure 11 also demonstrates how the main rotor wake consistently impinges on the front half of the tail rotor disk and, therefore, why the unsteadiness in the thrust coefficient of the high tail rotor at an advance ratio of 0.12 (as shown in Fig. 8) is dominated by oscillations at the blade passage frequency of the tail rotor.

Figures $10-12$ show that the coalescence of the main rotor tip vortices into a supervortex in the region upstream of the tail rotor is not, in itself, a necessary prerequisite for adverse interaction between the main and tail rotors of a conventional helicopter. Indeed, only the

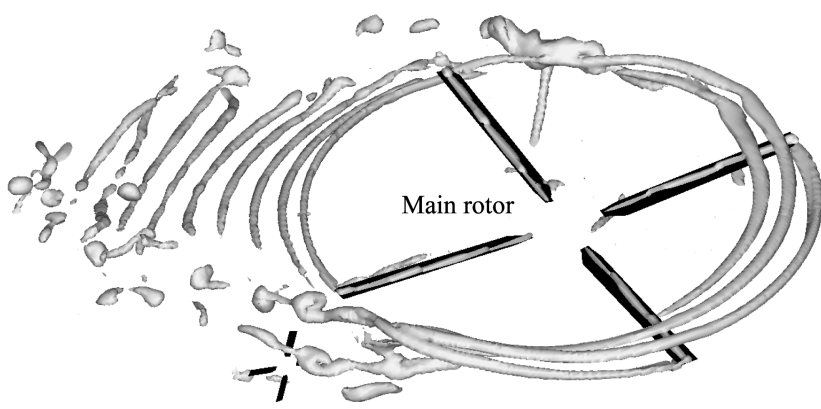

a) MR-TR system with a low tail rotor, $\mu=0.07$

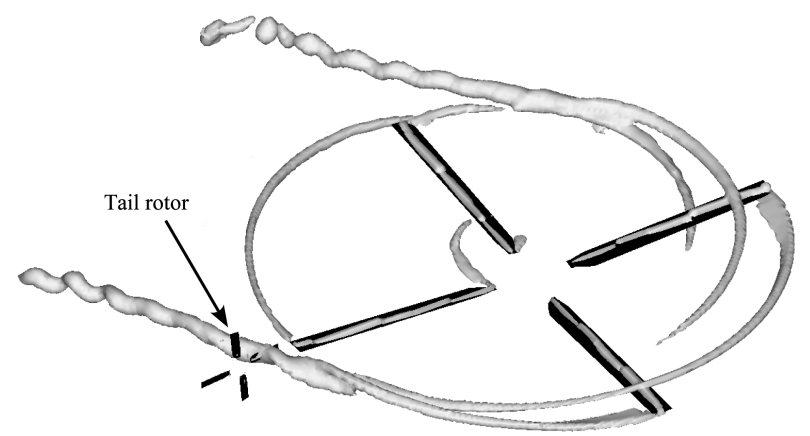

b) MR-TR system with a high tail rotor, $\mu=0.12$

Fig. 12 Highly resolved instantaneous snapshot of the wake of a topforward tail rotor operating with the main rotor in $60^{\circ}$ sideslip. character of the unsteadiness in the tail rotor thrust coefficient is influenced by whether the tail rotor interacts with a large coherent region of vorticity (or supervortex) or a cluster of smaller, but distinct, vortex filaments. The directionally dependent mode is caused only by the net interaction between the tail rotor and the vorticity within the main rotor wake.

Figure 13 shows the distribution of the blade aerodynamic loading, the resultant sectional velocity, and the local angle of attack on the low tail rotor when the MR-TR system operates at a pretransitional advance ratio. The data that are shown in Fig. 13a were obtained by simulating a top-aft tail rotor in isolation. Figure $13 \mathrm{~b}$ shows the difference between the blade loading, the sectional velocity, and the angle of attack that were developed on the top-aft tail rotor and that developed by the isolated tail rotor. Figure $13 \mathrm{c}$ shows data equivalent to those shown in Fig. 13b but, instead, for the difference between the top-forward tail rotor and the isolated tail rotor. The advance ratio of the MR-TR system was held constant in all of the simulations that are shown in Fig. 13. Although the distribution of aerodynamic loading, sectional velocity, and angle of attack exhibits some variability over the period of several tail rotor revolutions, the data that are shown in Fig. 13 for one complete revolution of the tail rotor are representative of the quasi-steady behavior of the rotor. Figure 14 shows equivalent distributions of the blade loading, the sectional velocity, and the angle of attack on the high tail rotor when the rotor system operates at an advance ratio of 0.12 .

The freestream velocity engenders an asymmetry in the aerodynamic loading, the sectional velocity, and the local angle of attack on the tail rotor blades, as shown in Fig. 13a. For the isolated tail rotor with a top-aft sense of rotation, that is shown in Figs. 13a and 14a, the tail rotor blades develop slightly more lift on the lower half of the rotor, as the blades advance into the freestream. Figure 13a shows that the loading on the blades as they traverse the upper half of the disk is reduced, as would be expected since the blades are retreating from the freestream. A comparison of Figs. 13a and 14a shows that the modest increase in the advance ratio of the system from 0.07 to 0.12 results in an increase in the asymmetry of the blade loading, the sectional velocity, and the local angle of attack over the tail rotor disk.

Figure 13b shows that when the MR-TR system operates at a pretransitional advance ratio, the aerodynamic loading on the blades of the top-aft tail rotor is significantly higher than that developed by the blades of the isolated tail rotor. In contrast, Fig. $13 \mathrm{c}$ demonstrates 


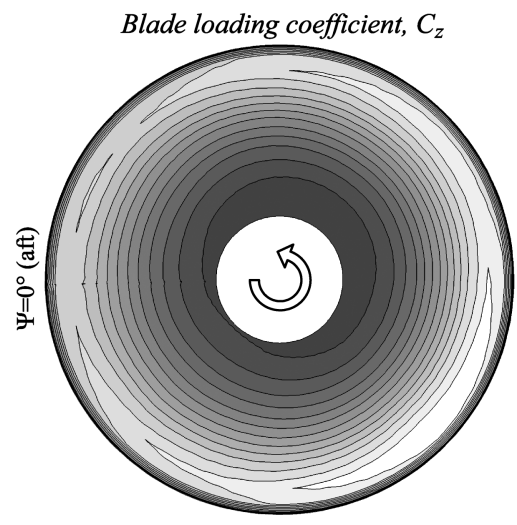

a) Isolated (top-aft) tail rotor

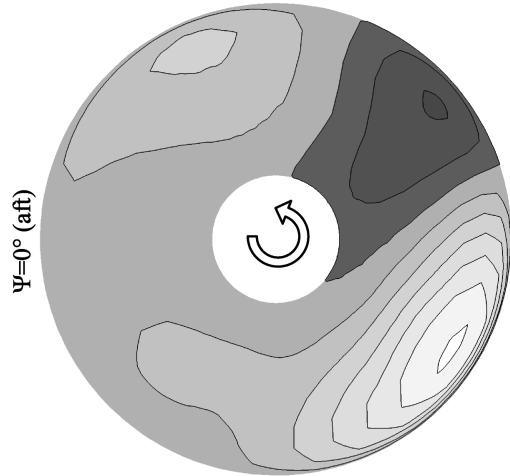

b) Top-aft tail rotor
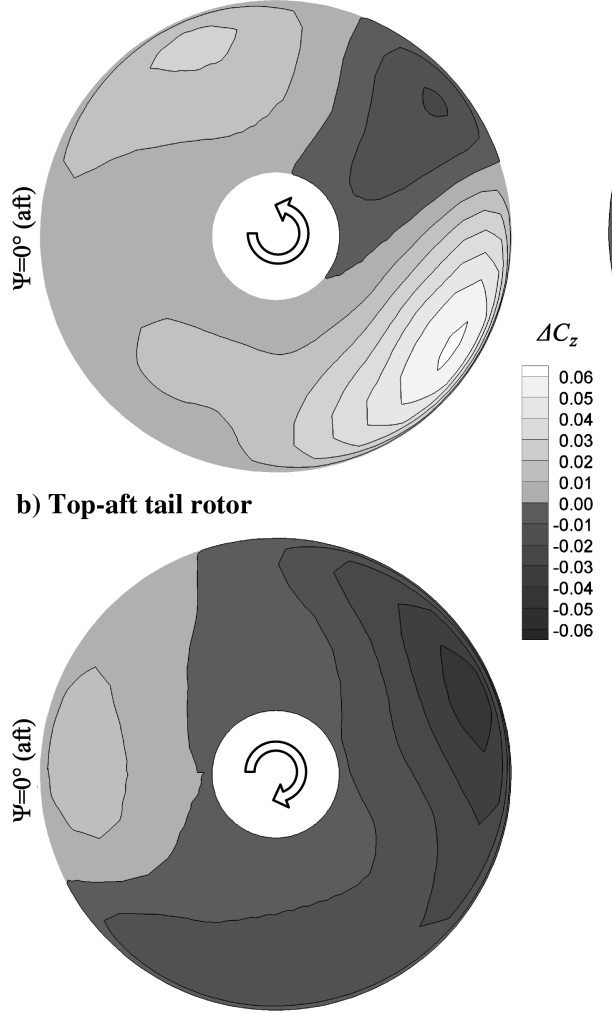

Resultant sectional velocity, $U$
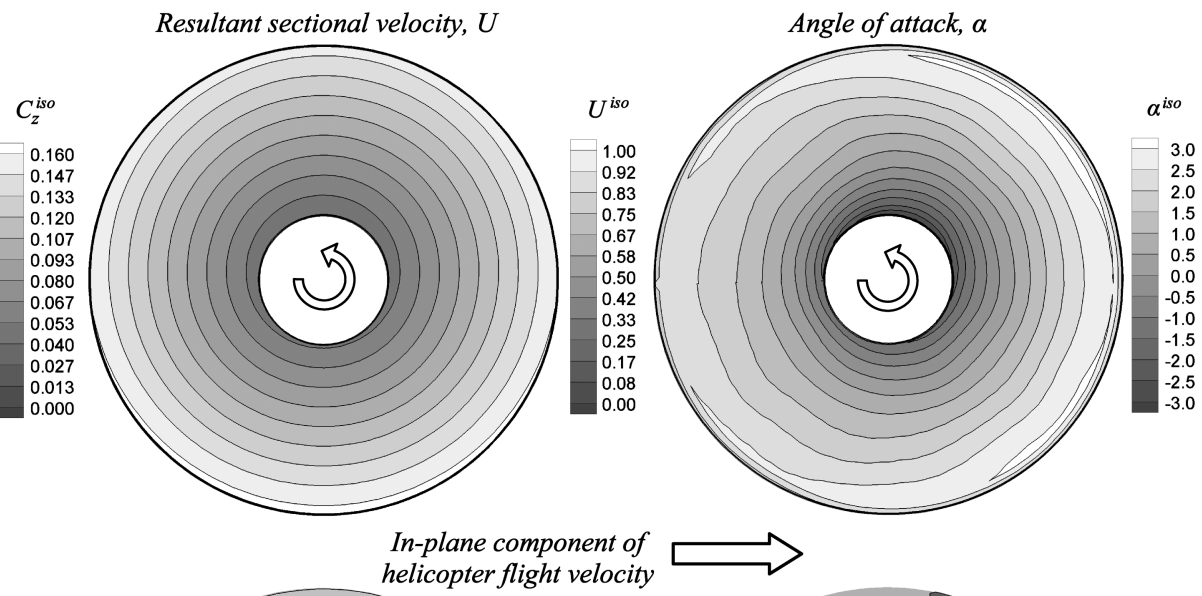
3.0
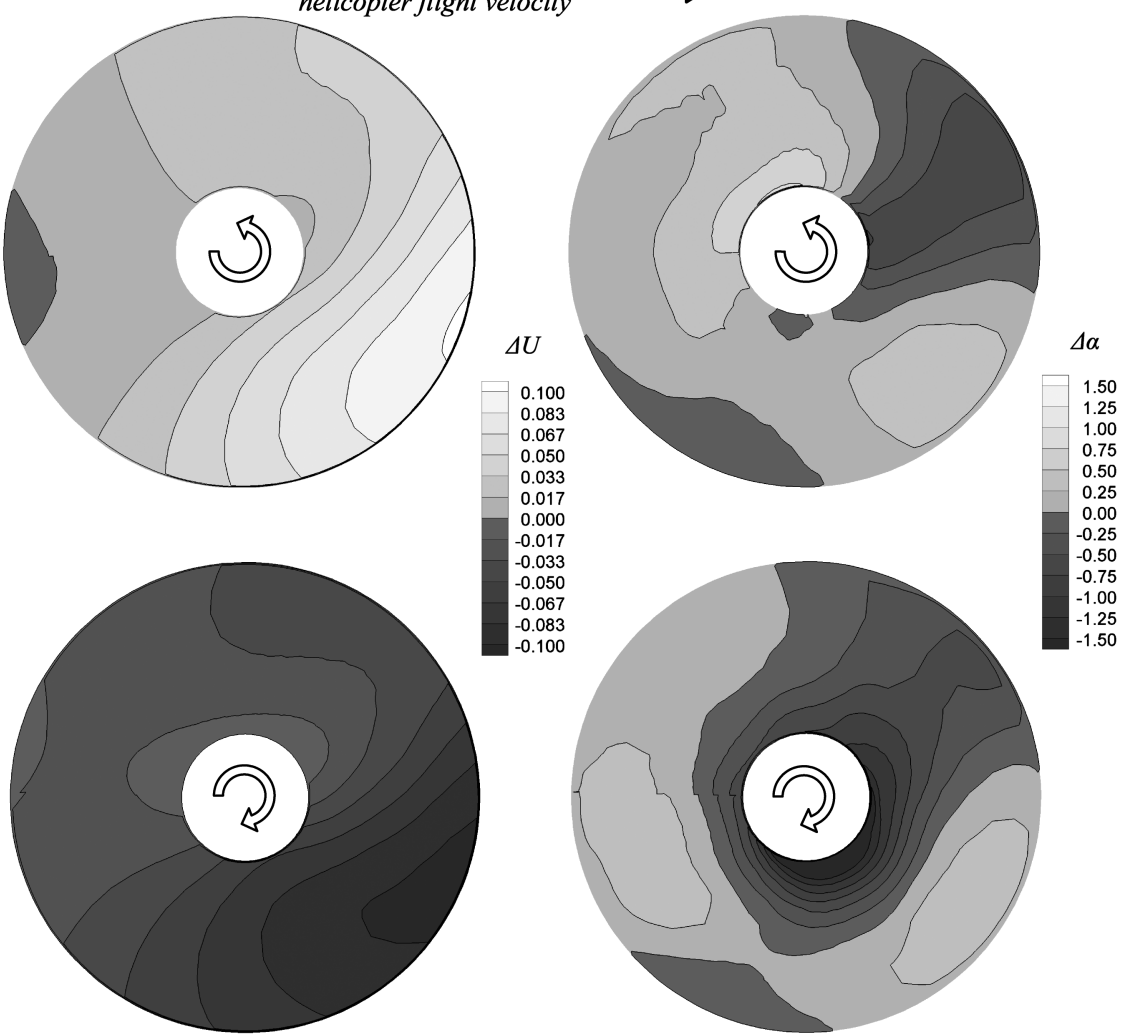

c) Top-forward tail rotor

Fig. 13 Representative distributions of blade loading coefficient (left), resultant sectional velocity (center), and local angle of attack (right) on an isolated top-aft tail rotor and on low tail rotors with opposing senses of rotation. Advance ratio $=0.07$.

that the blades of the top-forward tail rotor develop an aerodynamic loading coefficient that is significantly lower than that on the blades of the isolated tail rotor. The variation of the aerodynamic loading on both the top-aft and the top-forward tail rotors correlates with the thrust coefficient of each of the two rotor configurations that are shown in Fig. 3. The top-aft tail rotor develops, in general, a higher blade loading and thrust coefficient than the equivalent isolated tail rotor. In contrast, the blades of the top-forward tail rotor are, in general, more lightly loaded, and the rotor develops a lower thrust coefficient than the isolated tail rotor. Figure 14b shows that when the high tail rotor is configured with a top-aft sense of rotation and is simulated at a posttransitional advance ratio, there is a strong asymmetry in the aerodynamic loading of the blades when they are on opposing sides of the rotor disk. The blades develop significantly greater aerodynamic loading than the blades of the isolated rotor during the lower half of the rotor rotation, but they develop a considerably lower loading than the blades of the isolated tail rotor during the upper half of the rotor azimuth. The imbalance in the blade loading coefficient that is shown in Fig. 14b is consistent with the thrust coefficient data that are shown in Fig. 4, which shows that, even though the high tail rotor with a top-aft sense of rotation interacts with the main rotor, the net effect of this interaction is for the tail rotor to develop a thrust coefficient that is very similar to that of the equivalent isolated tail rotor. Figure $14 \mathrm{c}$ shows that the blades of the high tail rotor with a top-forward sense of rotation are considerably more lightly loaded than the blades of the isolated tail rotor, over most of the tail rotor disk.

Figure $13 \mathrm{~b}$ indicates that the increase in the aerodynamic loading on the blades of the top-aft tail rotor is closely related to the increase in the resultant flow velocity at the blades when compared with that experienced by the blades of the isolated tail rotor. Importantly, the increase in the sectional velocity at the blades of the top-aft tail rotor appears to be the result of the rotational motion of the blades within the downwash that is associated with the main rotor wake. Indeed, Fig. 13b shows no clear impingement on the tail rotor of a large coherent vortical structure. Rather, when the MR-TR system translates at pretransitional advance ratio, the net influence that the distinct but small regions of vorticity, shown in Fig. 12a, have on the sectional velocity at the tail rotor blades is diffuse. Figure $13 \mathrm{c}$ shows that, in contrast to the top-aft tail rotor, the blades of the top-forward 
tail rotor encounter lower sectional velocity over most of the rotor disk. Both Figs. 13b and 13c suggest that the aerodynamic loading on the blades of the tail rotor is moderated, however, by a notable reduction in the local angle of attack that is experienced by the blades when they are in the upper forward quadrant of the rotor. This reduction in the angle of attack of the blades is caused by an increase in the inflow at the tail rotor as it is immersed within the downwash from the main rotor.

Figures $14 \mathrm{~b}$ and $14 \mathrm{c}$ demonstrate clearly that the influence on the sectional velocity at the blades of the high tail rotor of its aerodynamic interaction with the main rotor is strongly dependent on the sense of rotation of the tail rotor. Figure $14 \mathrm{~b}$ shows that the interaction of the tail rotor with the large and coherent region of vorticity (supervortex) that develops downstream of the main rotor results in almost the entire area of the top-aft tail rotor experiencing higher sectional velocities than those that are experienced by the isolated tail rotor. In contrast, Fig. $14 \mathrm{c}$ shows that the sectional velocity at the blades of the top-forward tail rotor is reduced in comparison to the isolated tail rotor over most of the rotor disk. Figure $12 \mathrm{~b}$ shows that, when the MR-TR system operates at an advance ratio of 0.12 , the supervortex impinges inboard on the tail rotor disk, just forward of the rotor axis. This interaction manifests as a small region on both the top-aft and the top-forward tail rotors where the difference in the sectional velocity compared with the isolated tail rotor is opposite to the trend that is exhibited elsewhere on the rotor disk. Figures $14 \mathrm{~b}$ and $14 \mathrm{c}$ also show that both the top-aft and the top-forward tail rotor experience significant reductions in the angle of attack of the blades over a large region of the rotor disk when compared with the angle of attack of the blades of the isolated tail rotor. This reduction in angle of attack is caused by the increased inflow through the rotor that is associated with the immersion of the high tail rotor within the main rotor wake as the rotor system translates at posttransitional advance ratios.

The quite different structure of the main rotor wake as it impinges upon the tail rotor that is shown in Fig. 12 demonstrates that the existence of a large, coherent supervortex is not an essential prerequisite for MR-TR interaction to have an adverse effect on the thrust that is developed by tail rotors with a top-forward sense of rotation. The advance ratios over which the directionally dependent mode can manifest, and the associated morphology of the main rotor

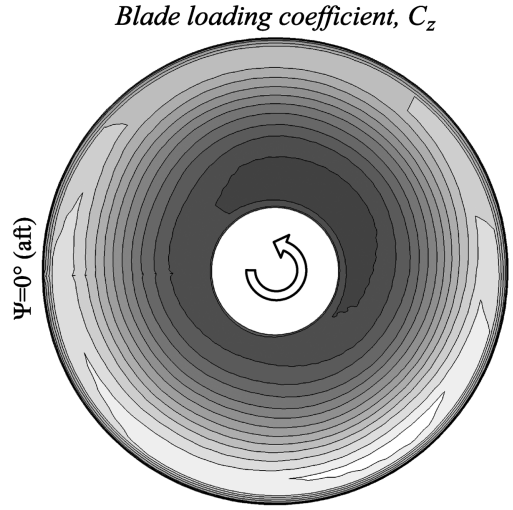

a) Isolated (top-aft) tail rotor

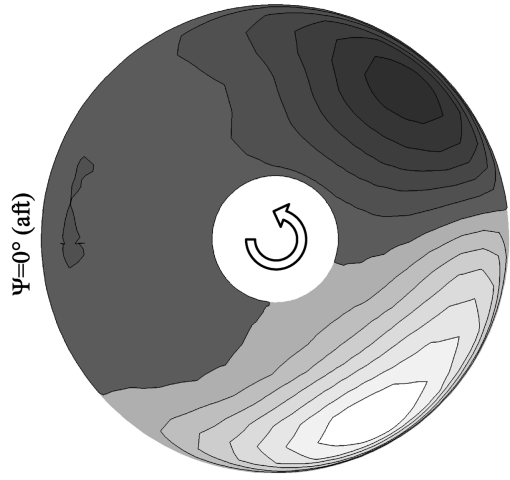

b) Top-aft tail rotor

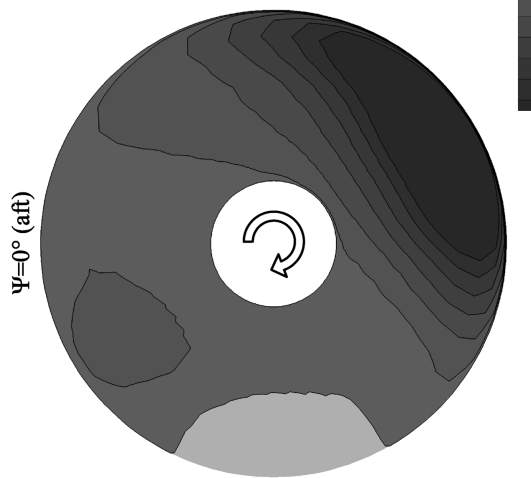

Resultant sectional velocity, $U$
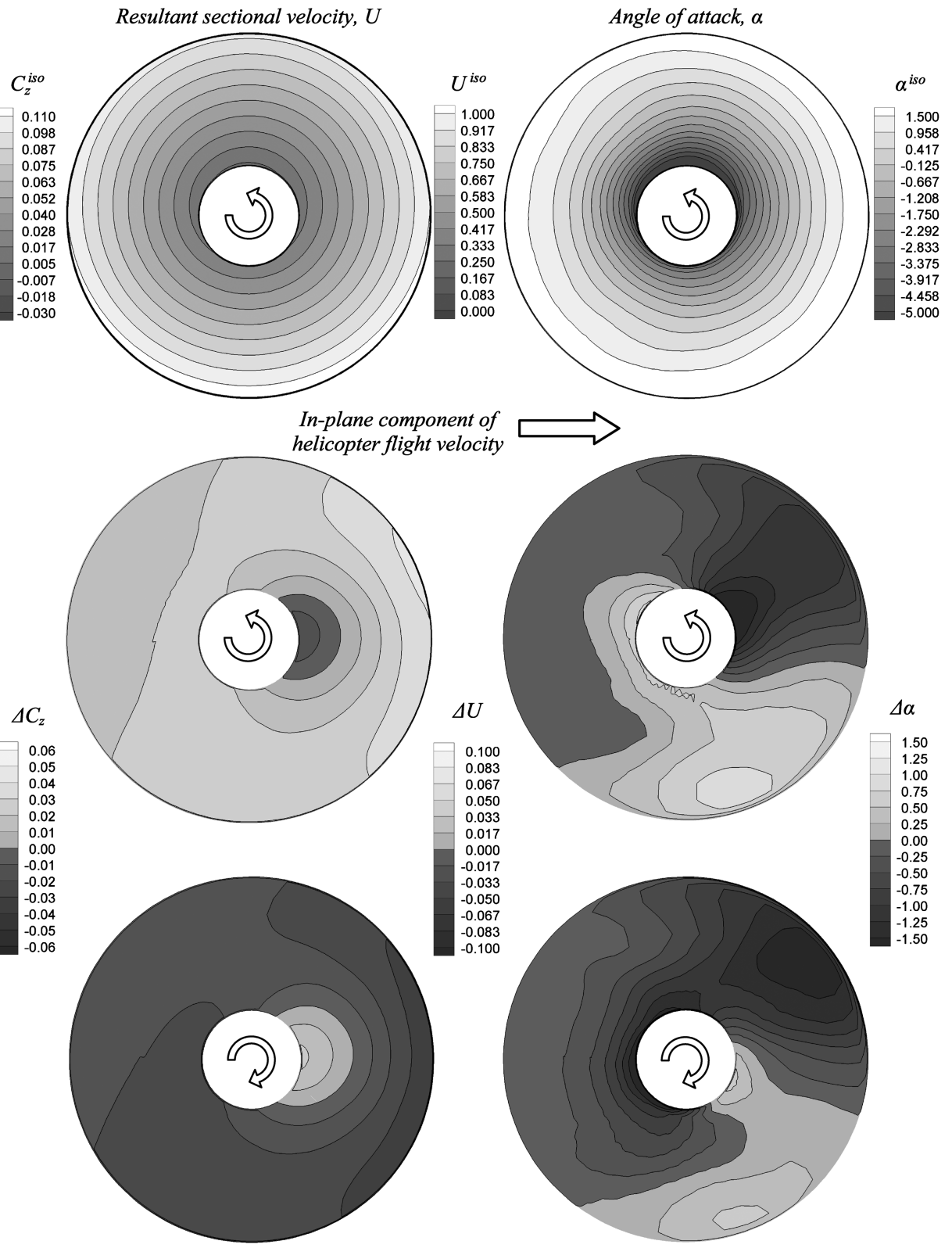
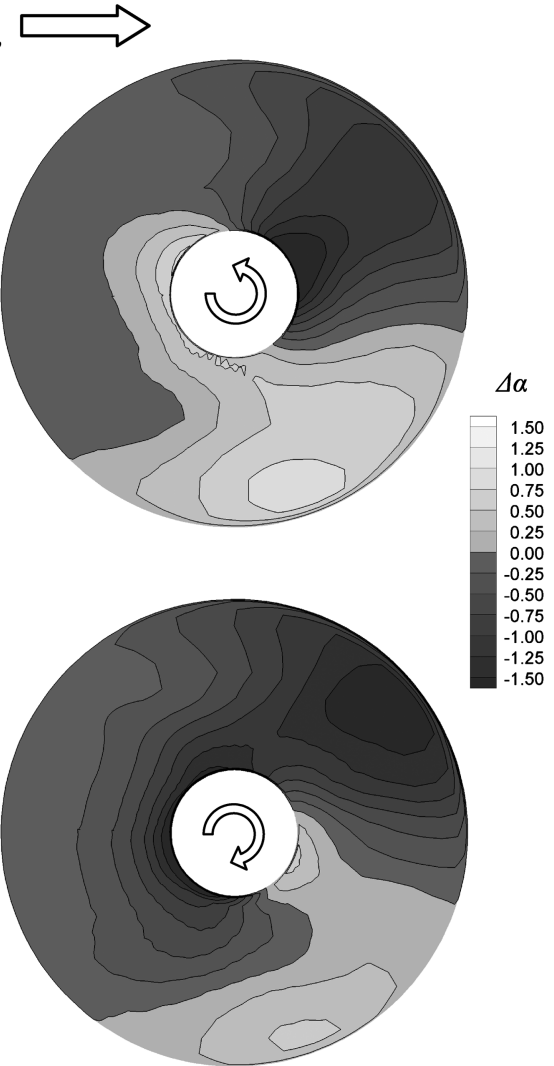

c) Top-forward tail rotor

Fig. 14 Representative distributions of blade loading coefficient (left), resultant sectional velocity (center), and local angle of attack (right) on an isolated top-aft tail rotor and on high tail rotors with opposing senses of rotation. Advance ratio $=0.12$. 
wake, vary considerably, depending on the vertical position of the tail rotor. At low flight speeds, where the structure of the main rotor wake is pretransitional, the net effect of the cluster of individual vortices that propagate toward the tail rotor is to significantly reduce the local dynamic pressure at the blades of the top-forward tail rotor. In contrast, a tail rotor with a top-aft sense of rotation can experience a substantial increase in local dynamic pressure and thrust due to constructive interference, whereby the velocity field that is induced by the main rotor wake adds to the resultant velocity at the blades that is produced by the rotation of the tail rotor. At higher, posttransitional advance ratios, the structure of the main rotor wake is such that a large coherent supervortex forms downstream of either side of the main rotor wake. In this portion of the flight envelope of the helicopter, the tail rotor can also operate within the directionally dependent mode but as a result of its interaction with a large coherent region of rotational flow that combines with the rotation of the tail rotor to influence its performance.

\section{Conclusions}

The tail rotor of a single main rotor helicopter can experience a significant reduction in thrust when the aircraft operates in crosswind flight at a high angle of sideslip. In practice, the pilot must increase the collective pitch of the tail rotor blades to maintain the desired yaw attitude of the aircraft, but the implication is that the margin for directional control of the helicopter will be reduced significantly as a result. A simplified helicopter model that was composed of only a single main rotor and tail rotor has been simulated when operating at a range of flight speeds with a $60^{\circ}$ angle of sideslip using the VTM.

The tail rotor has been shown to operate within two distinct modes that are created by its interaction with the wake that is induced by the main rotor. In the first mode, the performance of the tail rotor is not affected by its sense of rotation. In the second, or directionally dependent, mode, the thrust that the tail rotor develops when operated at constant collective pitch is considerably higher when the tail rotor has a top-aft sense of rotation compared with when its blades travel forward at the top of the disk. The range of helicopter advance ratios over which the tail rotor operates within the directionally dependent mode has been shown to be dependent on the vertical location of the tail rotor. In general, the directionally dependent mode exists at higher flight speeds when the tail rotor is mounted in a higher position with respect to the main rotor. When the tail rotor is located in a relatively low position, the directionally dependent mode exists only within a finite range of flight speeds. At advance ratios beyond those associated with the directionally dependent mode, the performance of the tail rotor is insensitive to its direction of rotation.

As the main rotor wake impinges on the tail rotor, the rotational velocity of the tail rotor combines with the induced rotation within the wake to strongly influence the sectional velocity and the angle of attack that the tail rotor blades experience as they rotate. As the rotation of the top-aft tail rotor opposes the vorticity within the main rotor wake, the directionally dependent mode manifests as a higher resultant flow speed at the blades compared with that at the blades of the isolated tail rotor. In contrast to the findings of previous studies, the existence of the directionally dependent mode does not, in all cases, require the impingement of the coherent supervortex that is developed downstream of the main rotor. Indeed, at relatively low advance ratios, in which the main rotor wake largely retains its tubular form, the performance of the tail rotor can be influenced by the net velocity field that is associated with a cluster of smaller, individual vortices that have not coalesced to form a supervortex. At higher flight speeds, in which the main rotor wake has transitioned into a more flattened structure, coherent supervortices are created by the coalescence of the individual vortices that are trailed behind the tips of the main rotor blades. If these supervortices impinge on a significant portion of the tail rotor, they induce an increase in the resultant flow velocity at the blades of the tail rotor when it rotates in the top-aft sense. In contrast, the rotational flow that is induced by the supervortices reduces the sectional velocity at the blades of the top- forward tail rotor and, consequently, reduces the thrust that the topforward tail rotor develops.

\section{References}

[1] Lynn, R. R., Robinson, F. D., Batra, N. N., and Duhon, J. M., "Tail Rotor Design Part 1: Aerodynamics," Journal of the American Helicopter Society, Vol. 15, No. 4, 1970, pp. 2-15. doi:10.4050/JAHS.15.2

[2] Johnston, J. F., and Cook, J. R., "AH-56A Vehicle Development," Proceedings of the American Helicopter Society 27th Annual Forum, AHS International, Alexandria, VA, 4-6 May 1971, pp. 1-10.

[3] Batra, N. N., "Results of a Tail Rotor Direction-of-Rotation Test," Journal of the American Helicopter Society, Vol. 15, No. 3, 1970, pp. 43-44.

[4] Leishman, J. G., Principles of Helicopter Aerodynamics, 2nd ed., Cambridge Univ. Press, New York, 2006.

[5] Prouty, R. W., "The Tail Rotor in the Vortex Ring State: A Mystery," Vertiflite, 2002, pp. 16-17.

[6] Huston, R. J., and Morris, C. E. K., "A Note on a Phenomenon Affecting Helicopter Directional Control in Rearward Flight," Journal of the American Helicopter Society, Vol. 15, No. 4, 1970, pp. 38-45. doi:10.4050/JAHS.15.38

[7] Ellin, A. D. S., "Lynx Main Rotor/Tail Rotor Interactions: Mechanisms and Modelling," Proceedings of the Institution of Mechanical Engineers, Part G, Journal of Aerospace Engineering, Vol. 208, No. 2, 1994, pp. 115-128. doi:10.1243/PIME_PROC_1994_208_261_02

[8] Kaynes, I. W., Preatoni, G., Visingardi, A., Tino, N., Arzoumanian, C., Kampa, K., Hermans, C., Renier, B. S. M., Tchen-Fo, F., and Bettschart, N., "HELIFLOW Pitch-Up and Quartering Flight Experiments," Proceedings of the 26th European Rotorcraft Forum, National Aerospace Lab. of the Netherlands, Amsterdam, 26-29 Sept. 2000, pp. 41.1-41.12.

[9] Markiewicz, R. H., Jones, A. F., Marshall, R. J., Thornton, K. R., Hill, S. P., and Eckford, D. J., "An Experimental Programme for the DRA Mach Scaled Model Rotor Test Rig," Proceedings of the 22nd European Rotorcraft Forum, Royal Aeronautical Soc., London, U.K., 17-19 Sept. 1996, pp. 19.1-19.13.

[10] Wiesner, W., and Kohler, G., "Tail Rotor Performance in Presence of Main Rotor, Ground, and Winds," Journal of the American Helicopter Society, Vol. 19, No. 3, 1974, pp. 2-9. doi:10.4050/JAHS.19.2

[11] Brown, R. E., "Rotor Wake Modeling for Flight Dynamic Simulation of Helicopters," AIAA Journal, Vol. 38, No. 1, 2000, pp. 57-63. doi: $10.2514 / 2.922$

[12] Brown, R. E., and Line, A. J., "Efficient High-Resolution Wake Modeling Using the Vorticity Transport Equation," AIAA Journal, Vol. 43, No. 7, 2005, pp. 1434-1443. doi: $10.2514 / 1.13679$

[13] Toro, E. F., "A Weighted Average Flux Method for Hyperbolic Conservation Laws," Proceedings of the Royal Society of London, Series A: Mathematical and Physical Sciences, Vol. 423, No. 1865, 1989, pp. 401-418. doi:10.1098/rspa.1989.0062

[14] Kim, H. W., and Brown, R. E., "A Rational Approach to Comparing the Performance of Coaxial and Conventional Rotors," Journal of the American Helicopter Society, Vol. 55, No. 1, Paper 012003. doi:10.4050/JAHS.55.012003, 2010.

[15] Kenyon, A. R., and Brown, R. E., "Wake Dynamics and rotor-fuselage Aerodynamic Interactions," Journal of the American Helicopter Society, Vol. 54, No. 1, Paper 012003. doi:10.4050/JAHS.54.012003, 2009.

[16] Kim, H. W., Kenyon, A. R., Duraisamy, K., and Brown, R. E., "Interactional Aerodynamics and Acoustics of a Hingeless Coaxial Helicopter with an Auxiliary Propeller in Forward Flight," The Aeronautical Journal, Vol. 113, No. 1140, 2009, pp. 65-78.

[17] Balch, D. T., "Experimental Study of Main Rotor/Tail Rotor/Airframe Interaction in Hover," Proceedings of the 39th American Helicopter Society Annual Forum, AHS International, Alexandria, VA, 911 May 1983, pp. 1-14.

[18] Fletcher, T. M., and Brown, R. E., "Main Rotor-Tail Rotor Interaction and its Implications for Helicopter Directional Control," Journal of the American Helicopter Society, Vol. 53, No. 2, 2008, pp. 125-138. doi:10.4050/JAHS.53.125 